\title{
Unraveling the Tangled Complexity of DNA: Combining Mathematical Modeling and Experimental Biology to Understand Replication, Recombination and Repair
}

\author{
S. Robic ${ }^{1 *}$, J.R. Jungck ${ }^{2}$ \\ ${ }^{1}$ Department of Biology, Agnes Scott College, Decatur, GA 30030, USA \\ ${ }^{2}$ Department of Biology, Beloit College, Beloit, WI 53511, USA \\ This paper is dedicated to the memory of Nicholas R. Cozzarelli
}

\begin{abstract}
How does DNA, the molecule containing genetic information, change its three-dimensional shape during the complex cellular processes of replication, recombination and repair? This is one of the core questions in molecular biology which cannot be answered without help from mathematical modeling. Basic concepts of topology and geometry can be introduced in undergraduate teaching to help students understand counterintuitive complex structural transformations that occur in every living cell. Topoisomerases, a fascinating class of enzymes involved in replication, recombination and repair, catalyze a change in DNA topology through a series of highly coordinated mechanistic steps. Undergraduate biology and mathematics students can visualize and explore the principles of topoisomerase action by using easily available materials such as Velcro, ribbons, telephone cords, zippers and tubing. These simple toys can be used as powerful teaching tools to engage students in hands-on exploration with the goal of learning about both the mathematics and the biology of DNA structure.
\end{abstract}

Key words: DNA topology, knot theory, linking number, mathematical manipulatives, supercoiling, undergraduate education, topoisomerases, writhe, twist, DNA replication, recombination and repair

AMS codes: 92C40, 54-01, 97U60

\footnotetext{
${ }^{*}$ Corresponding author. E-mail: srobic@agnesscott.edu
} 


\section{Introduction}

What happens to the double helical structure of the DNA as a cell undergoes processes such as replication, recombination and repair? The answer to this complicated question is often presented to undergraduate biology students using phrases such as:

- At each replication fork, an enzyme called "helicase" unwinds the two DNA strands [1].

- DNA helicase separates strands, and DNA topoisomerase alleviates coiling ahead of the fork [2].

- Helicase is an enzyme that untwists the double helix at the replication fork. This untwisting causes tighter twisting and strain ahead of the replication fork, and topoisomerase helps relieve the strain [3].

- Topoisomerase II travels along the DNA ahead of the replication fork removing positive supercoils [4].

- We have helicases unwinding the strands and topoisomerases allowing the seperation to proceed right along the chromosome by relaxing positive supercoiling [5].

While these phrases may make perfect sense to a seasoned DNA biochemist, such language can be confusing to undergraduate students. These descriptions allude to complex three dimensional structural changes which are difficult to fully grasp solely based on verbal descriptions. Biology textbooks almost never include any discussion of the underlying mathematics. What does it really mean to "separate strands", "alleviate coiling", "untwist the double helix" and "remove positive supercoils"? How is the structure of DNA affected locally and globally during all these transformations? What is the energy cost or gain of these processes?

Simple toys, made of inexpensive materials such as rubber bands, tygon tubing, ribbons and zippers, can be used to generate a different, more complex and more dynamic image of the three-R processes of DNA: replication, recombination and repair. Now, more than five decades after the discovery of the helical structure of DNA, we have a clearer understanding of the tangling and intertwining of DNA, and a deeper appreciation for the complex regulatory mechanisms that prevent cell death by hopeless DNA entanglement each time the DNA replicates, recombines or is repaired. By combining exploration of physical models in the context of mathematical concepts of DNA geometry and topology, we can engage the students in becoming active participants in interpretation of experimental laboratory results. Rather than just reading phrases such as "alleviating coiling" and "unzipping DNA" students can explore these processes through their own experimentation with toys and models. By introducing some basic concepts from the mathematical field of topology, our goal is to provide the students with tools necessary for quantitative exploration of various physical models. 


\section{How does DNA “unzip” during replication?}

Most undergraduate students are familiar with double helical structure of DNA, the molecule that encodes genes in its one-dimensional sequence. However, familiarity with the double helix and the sequence it encodes is only the first step in understanding the function and structure of DNA. Our goal is to provide the students with some necessary tools and concepts to help them explore additional levels of DNA structure.

The three dimensional structure of DNA is complex, compacted, and undergoes dynamic conformational changes during cellular processes such as replication, recombination and repair. The same forces that cause computer cables to become entangled also apply to long strings of DNA. Mathematical models, involving geometric and topological concepts, help shed light on these processes, and may be used to engage both mathematics and biology students in interdisciplinary exploration of DNA structure and function $[6,7,8,9]$.

Does DNA simply "unzip" in DNA replication, the process through which DNA makes copies of itself? A typical textbook image of DNA replication shows a static replication fork, with two strands indicating the strands of the double helix, which simply open into a V-shaped structure (Figure 1). Diagrams such as this one are powerful tools for introducing molecular processes, but such static two-dimensional images are usually not sufficient to engage a student in deeper analysis of the complex biological process of DNA replication. Toys and simple physical models, such as strings, tubing, Velcro, and zippers provide the students with opportunities for hands on explorations of DNA structure, and transformations it undergoes as it is replicated, recombined or repaired.

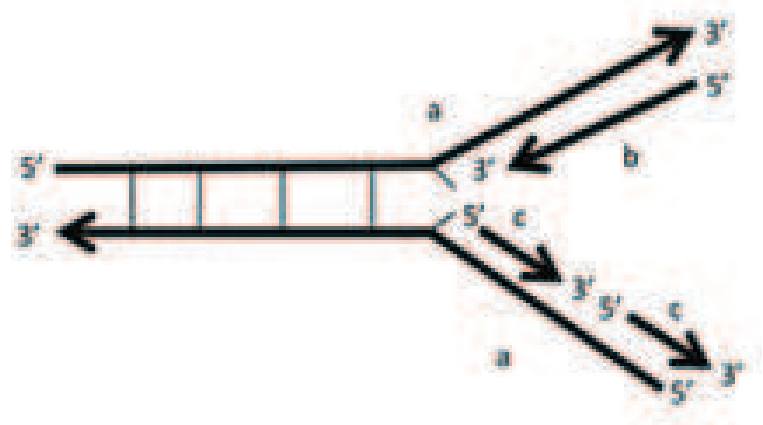

Figure 1: A typical textbook image of DNA replication fork oversimplifies a complex biological process. Positions 5' to 3' indicate the direction of each anti-parallel nucleic acid strand. The two long strands (a) represent the two strands of the original DNA molecule. The shorter strands (b and c) indicate the newly synthesized strands. The leading strand (b) is synthesized continuously, and the lagging strands (c) are synthesized as short Okazaki fragments, later joined by the enzyme ligase. 


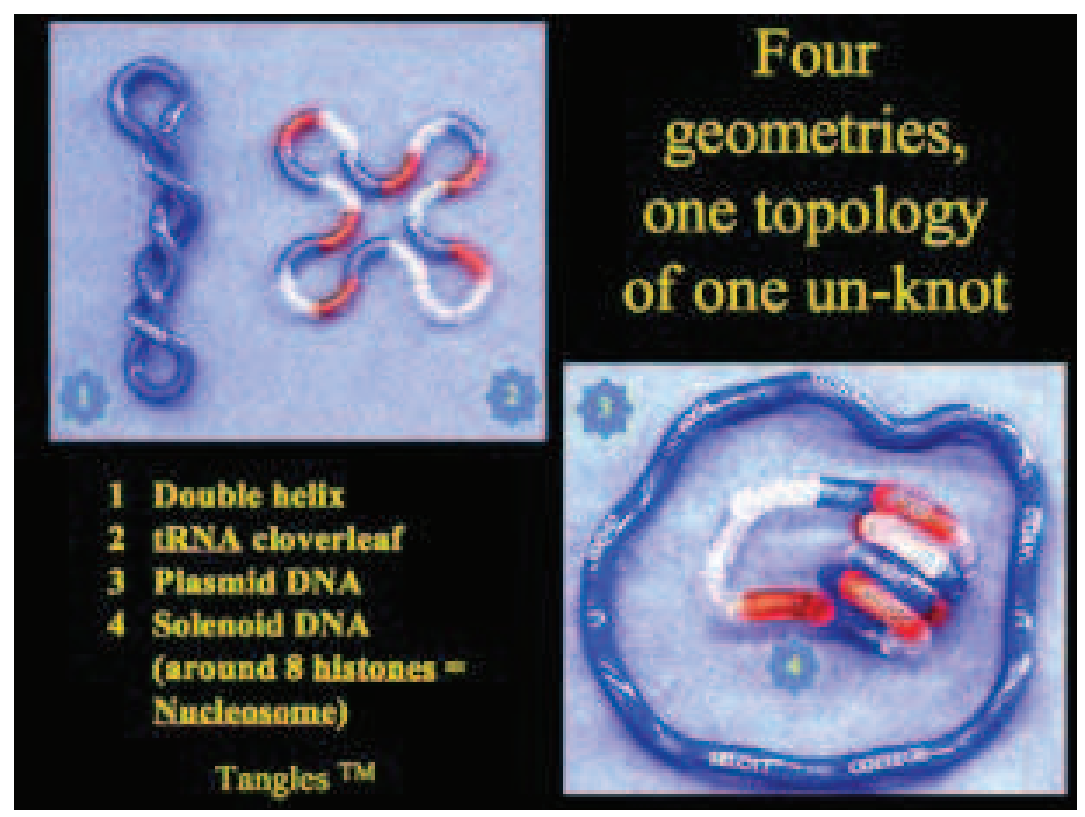

Figure 2: "Tangles" ${ }^{\mathrm{TM}}$ here are folded in four different ways commonly associated with mimicking DNA and RNA structures.

\section{Getting started with modeling}

As a preliminary activity, we recommend that students play with objects commonly used to demonstrate protein folding into alpha helices, beta-pleated sheets, random coils, etc. Some favorites include origami, "Toobers"TM " "Tangles" "TM (see Figure 2), and Rubik's snake ${ }^{\mathrm{TM}}$. In the latter case, students frequently compete to construct bird, dog, snake, turtle and other morphologies without breaking the "snake" but simply by folding in different ways. This experience helps them understand the combinatorial explosion of geometric conformers with identical topologies before tackling topology per se. Tim Herman's and Jacqueline Roberts' colleagues review some advantages of "the usefulness of physical models of molecular structures ... in capturing the interest of students ... [and] stimulat[ing] more sophisticated questions in the minds of students ..."[10, 11].

\section{DNA is not a Möbius Strip}

How does a topoisomerase cut relaxed DNA sufficiently such that the antiparallel complementary strands are separated far enough apart that each can be used as a template for synthesis on a new antiparallel complementary strand? Could DNA achieve this by adopting a Möbius strip structure as a step in this process? Let us begin by demonstrating an intuitive model that might lead to more problems rather than solve this dilemma if it were the way that "unwindases" worked. Students can use zippers to model the process of DNA unwinding. Sewing a ribbon of one color to one side of the zipper, and a different colored ribbon to the other side of the zipper allows the two stands to be distinguished. 
One of the most common topological problems to which students are exposed is that by introducing a half twist onto a ribbon one constructs a one-sided surface (a Möbius Strip) instead of two (Figure 3a). However this is not a valid possibility for DNA as the two ends connected after a half-twist abut two antiparallel strands against one another with opposing polarity, thus interfering with the 5' to 3' continuum imposed by chemical bonding in DNA. One feature of Möbius Strips usually highlighted is that if you cut them in half longitudinally, one gets one long band (Figure 3b). What happens for the minimal structure of a DNA double helix? Let us introduce one full twist (Figure 3c) into a bi-colored ribbon model of DNA; for such a structure, the polarity is preserved: $\left(5^{\prime} \rightarrow 3^{\prime}\right.$ abuts $\left.5^{\prime} \rightarrow 3^{\prime}\right)$ and $\left(3^{\prime} \leftarrow 5^{\prime}\right.$ abuts $\left.3^{\prime} \leftarrow 5^{\prime}\right)$. When this single twisted DNA is cut in half longitudinally, the two halves of the zippered molecule become two interlocked loops (Figure 3d). So even if the cell had separated the two strands and duplicated them, now the two daughter double helices could not simply be dispersed into the two daughter cells unless the interlocked strands were broken and rejoined individually.

For those interested in pursuing such types of cuts a series of nice Youtube videos are available from an author identified as "kitefrog" [12]. Eugene Babaev "Intuitive Chemical Topology Concepts" introduces corresponding graph theory by covering the multigraph representations of such surfaces, cuts, and circuits [13].

Not only is this exercise somewhat surprising to some students, the interlocked rings can be used to illustrate that without a topoisomerase, a cell would be in trouble because the unzipped and duplicated DNA strands wouldn't be able to move to the two daughter cells during cytokinesis. In Figure 4, students illustrate this problem with a much larger model.

\section{Dissecting a Coiled Coil}

The most common physiological structure of DNA is a right-handed double helix, also known as the B-form DNA. It consists of the two strands of DNA intertwined every 10.5 base pairs. This right-handed double helix can be thought of as a coil. The coil is itself further coiled to form structures known as supercoils, i.e., coiled coils $[14,15]$. Because supercoiling introduces additional levels of complexity to DNA structure, it is important how this complex structure can reproduce itself, and how the structure affects repair and recombination processes. A long zipper twisted around itself and joined at the ends with velcro can serve as a model of a supercoiled circular DNA (Figure 5).

Concepts from the fields of topology and geometry can be used to describe and quantify higher order DNA structures, such as supercoiling. Such concepts can be made easily accessible to undergraduate students through the use of physical models. Students' intuitive understanding of geometry is generally close to its precise mathematical definition. Objects of different shapes and/or sizes are geometrically different. For example, a student will typically understand that a sphere, a cube and a pyramid have different geometries. Similarly, straight and bent linear DNA molecules have different geometries.

Topology, on the other hand, is a broader term than geometry. Two objects are topologically equivalent if one can be continuously deformed into the other, i.e., if we can change from one 


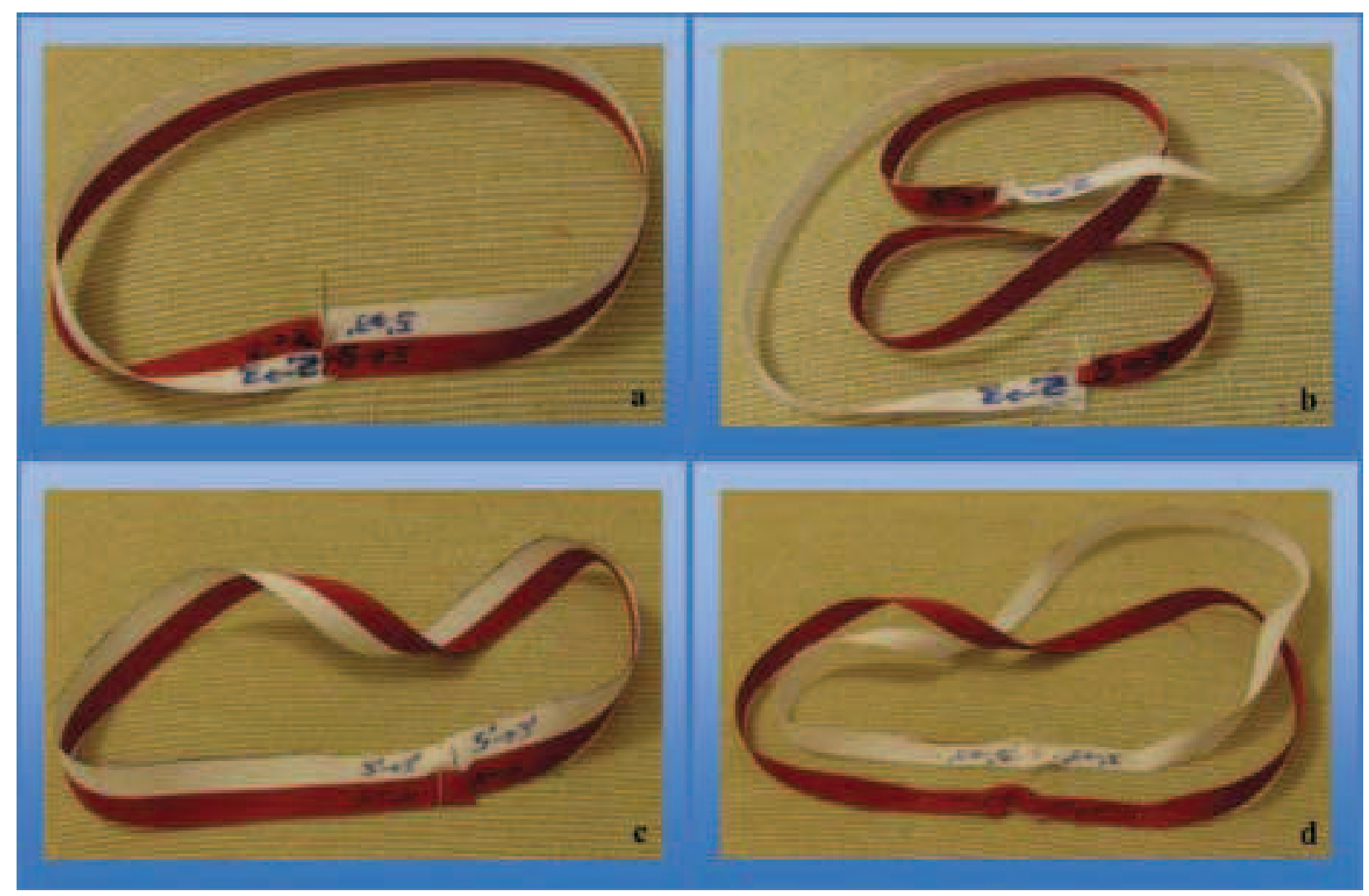

Figure 3: (a) Möbius Strip ribbon model of DNA shown not to be possible because a half-twist abuts two anti-parallel strands against one another with opposing polarity: 5' end of one strand is now joined with the 5' end of another strand, and 3' end of one strand is joined with 3' end of the other strand. Such bonding is not chemically possible in the DNA. 5' carbon of one deoxyribose can only be linked to the 3' carbon of another. (b) If you cut a Möbius Strip in half longitudinally, one gets one long band. (c) If, instead, one full twist is introduced into this bi-colored ribbon model of a minimal structure of a DNA double helix; the polarity is preserved: $\left(5^{\prime} \rightarrow 3^{\prime}\right.$ abuts $\left.5^{\prime} \rightarrow 3^{\prime}\right)$ and $\left(3^{\prime} \leftarrow 5^{\prime}\right.$ abuts $\left.3^{\prime} \leftarrow 5^{\prime}\right)$. (d) When this single twisted DNA is cut in half longitudinally, one gets two short bands.

shape to the other without introducing any breaks. For example, a cube made out of play dough can be continuously deformed into a sphere, without ever puncturing the cube. On the other hand, a sphere cannot be continuously deformed into a torus (donut-shaped solid). If we want to perform such a deformation, we have to puncture the sphere, which means that a donut and a sphere are topologically distinct. Circular and linear DNA are the simplest examples of different DNA topologies. Circular DNA topology can manifest itself in many different geometries (Figures 2 and 6).

Both geometry and topology provide us with valuable vocabularies to describe complex DNA structures. There are two major classes of DNA supercoiling: plectonemic and solenoidal (Figure 8)[15]. Plectonemic, or unrestrained supercoiling occurs on circular bacterial chromosomes; 

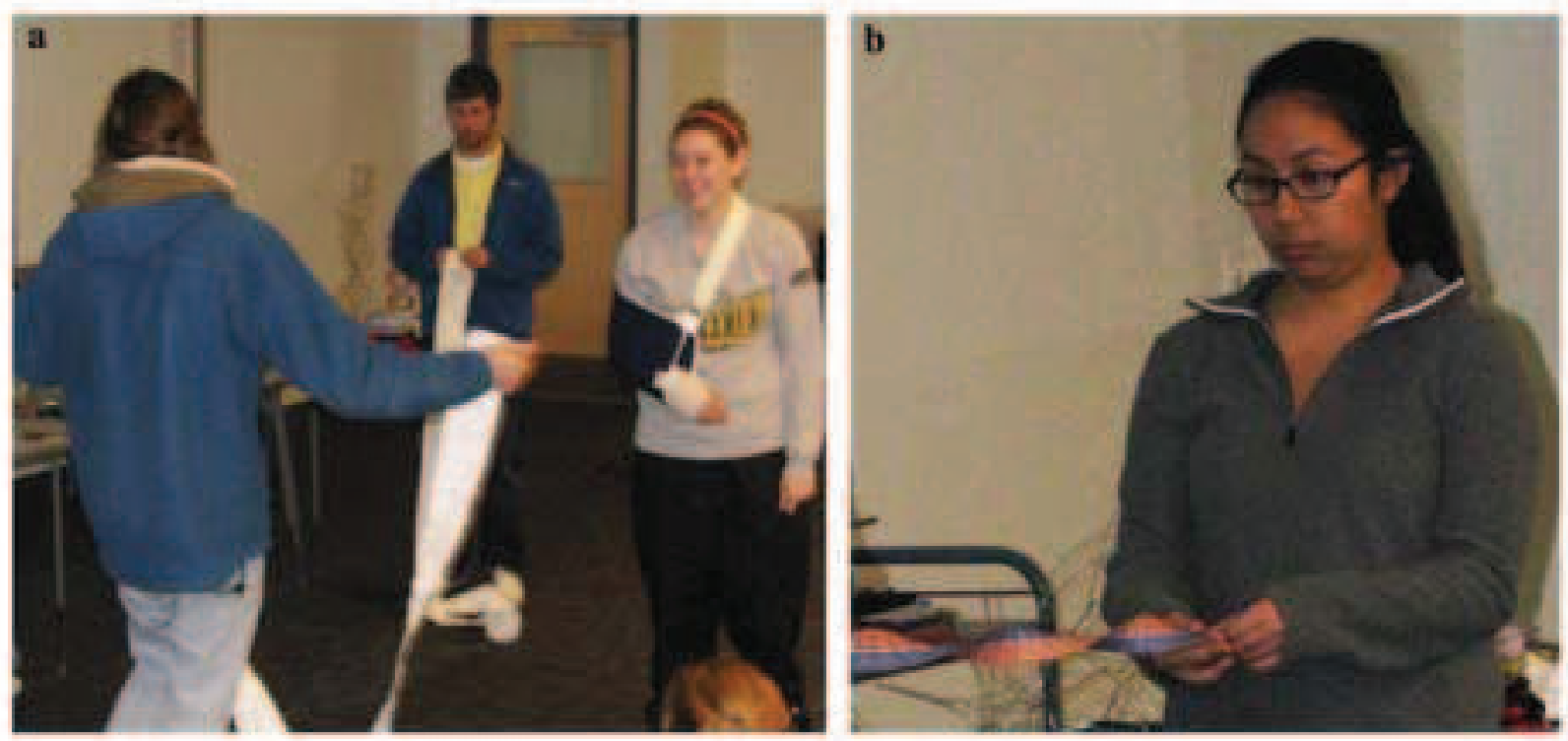

Figure 4: (a) Beloit College students Geoffrey Pratt, Kellen Sullivan, and McKenzie Falker try to separate a pair of strands like in Figure 3d except in this case the two strands are connected to one-another by face to face Velcro ${ }^{\mathrm{TM}}$ - as they pull the two loops formed, they develop a tight knot at the joint where the two strands contact one another. (b) Beloit College student Jourdan Posner twists a bi-color ribbon before cutting the two strands apart.

whereas soilenoidal supercoiling is restrained by bound protein structures and can be found in eukaryotic DNA, wrapped around the histones (Figure 8) [16].

Both of these forms of supercoiling can be modeled in a classroom (Figures 6 and 7). A pair of shoe-laces or ribbons, forced to wind around each other prior to tying two ends will adopt a plectonemic structure. A soilenoidal supercoil can be modeled using an empty paper towel roll or a graduated cylinder as a histone scaffold (Figure 6c). Such easy-to-build physical models are powerful tools for demystifying higher-order structures of DNA. Physical manipulations, such as stretching and twisting, allow students to grasp what happens when such structures are challenged by further strain, such as the strain introduced by various enzymes interacting with the DNA (Figure 7).

\section{Supercoiling}

To understand the convention of positive and negative supercoiling, the positive or negative sign of direction is illustrated in Figure 9.

After a closer look at supercoiled structures, it becomes apparent that there are two levels of coiling within each supercoil. One of them is coiling of the strands (DNA strands, or shoe laces) around the central axis (superhelical axis), and the other one is coiling of the superhelical axis itself. Coiling of strands around the axis is quantified by the twist (T), while coiling of the axis 

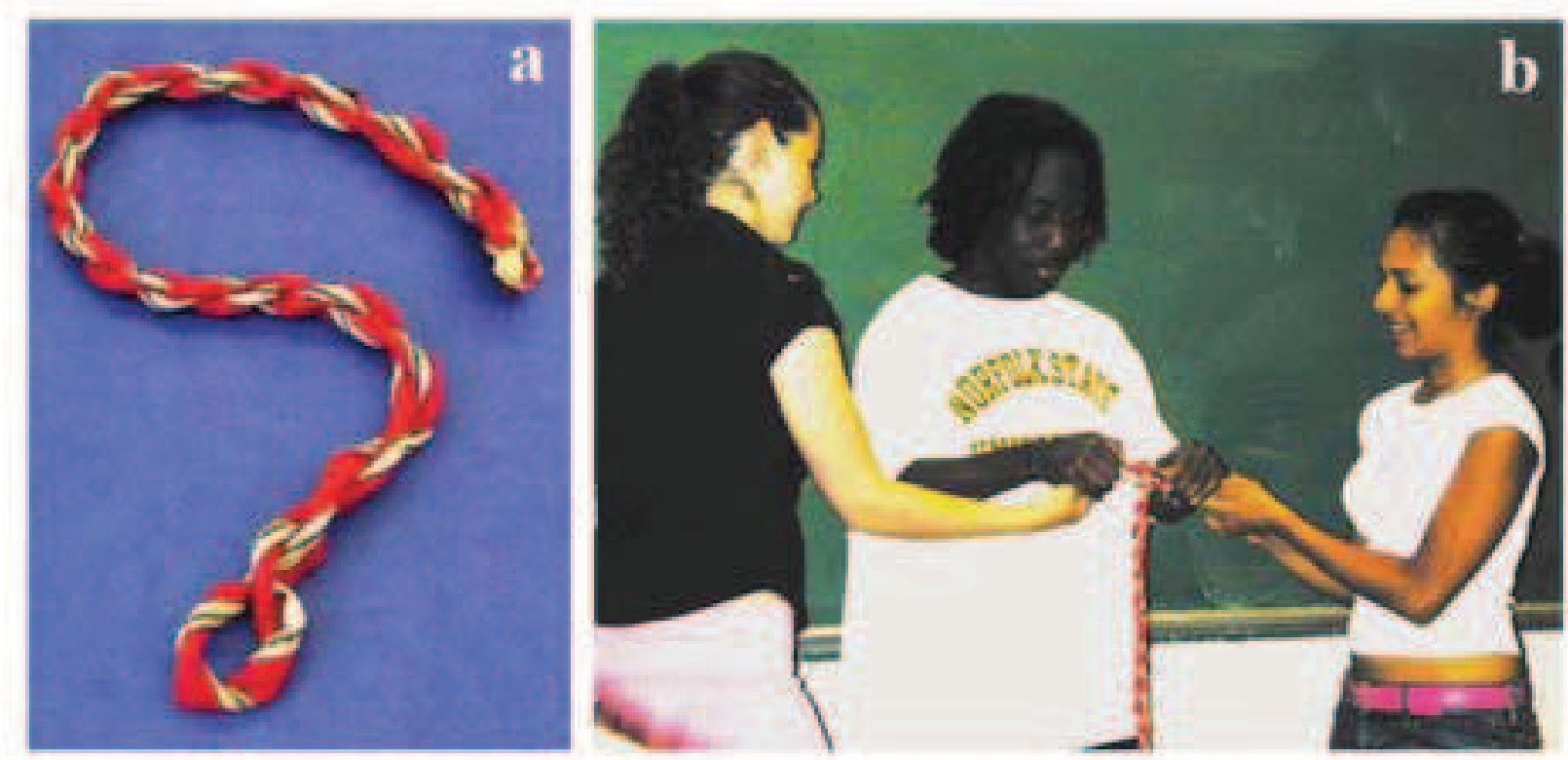

Figure 5: (a) A coiled zipper model with Velcro on both ends to convert a "linear chromosome" into a "circular chromosome or plasmid" can be used to demonstrate how difficult it is to "unzip" a supercoiled DNA molecule in replication. Students who try to unzip this tightly coiled zipper realize that it is almost impossible to do it without a great deal of effort. (b) Three students who were participants in the Mathematical and Theoretical Biology Institute at Los Alamos National Labs led by Professor Carlos Castillo-Chavez of Cornell University (he and the program have since moved to Arizona State University) are shown here working with DNA zipper models.

itself is measured by writhe (W) $[14,20,21]$. The two parameters are related to each other and to the topological parameter linking number $(\mathrm{L})$, which will be discussed in the next section.

\section{Linking numbers}

Linking number can be defined as the number of times one strand crosses the other when DNA is placed flat onto a plane. Linking number can be assigned to any closed DNA structure. The number L must be an integer, and stays the same for any topology of DNA. Therefore, the linking number is considered a topological invariant for a molecule of DNA, unless DNA is cut and its strands are cut and rejoined after being rotated around each other.

Linking number can be determined from two oriented curves by considering a projection of the two curves onto a two-dimensional plane. This is directly applicable to studies of DNA because, due to its chemical makeup, we can assign a direction $\left(5^{\prime} \rightarrow 3^{\prime}\right)$ to each DNA strand. To do this we draw a projection of two linked curves by interrupting the under-passing segment and leaving the over-passing segment continuous. To each crossing, also known as a node, we can assign a value of +1 or -1 , according to the convention shown in Figure 10. Once we assign +1 or -1 to all 


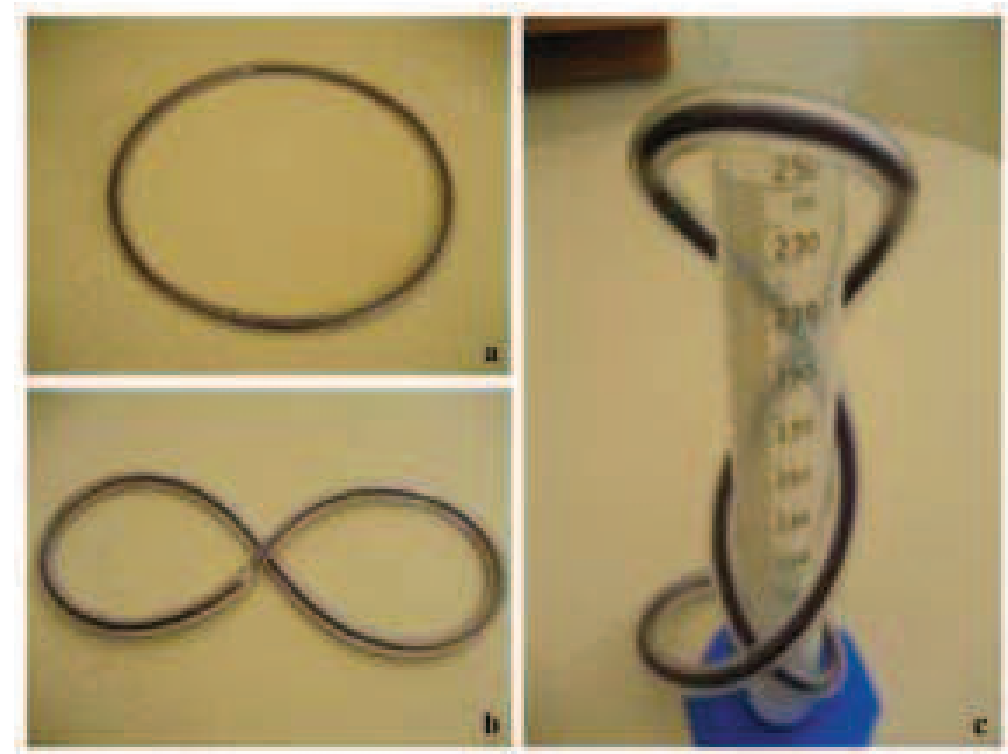

Figure 6: Tygon tubing models of three different DNA geometries of circular DNA: (a) uncoiled circular, (b) circular with one coil, and (c) circular wrapped around a cylindrical object. Each of the geometries shown above can be easily transformed into another without introducing any breaks.
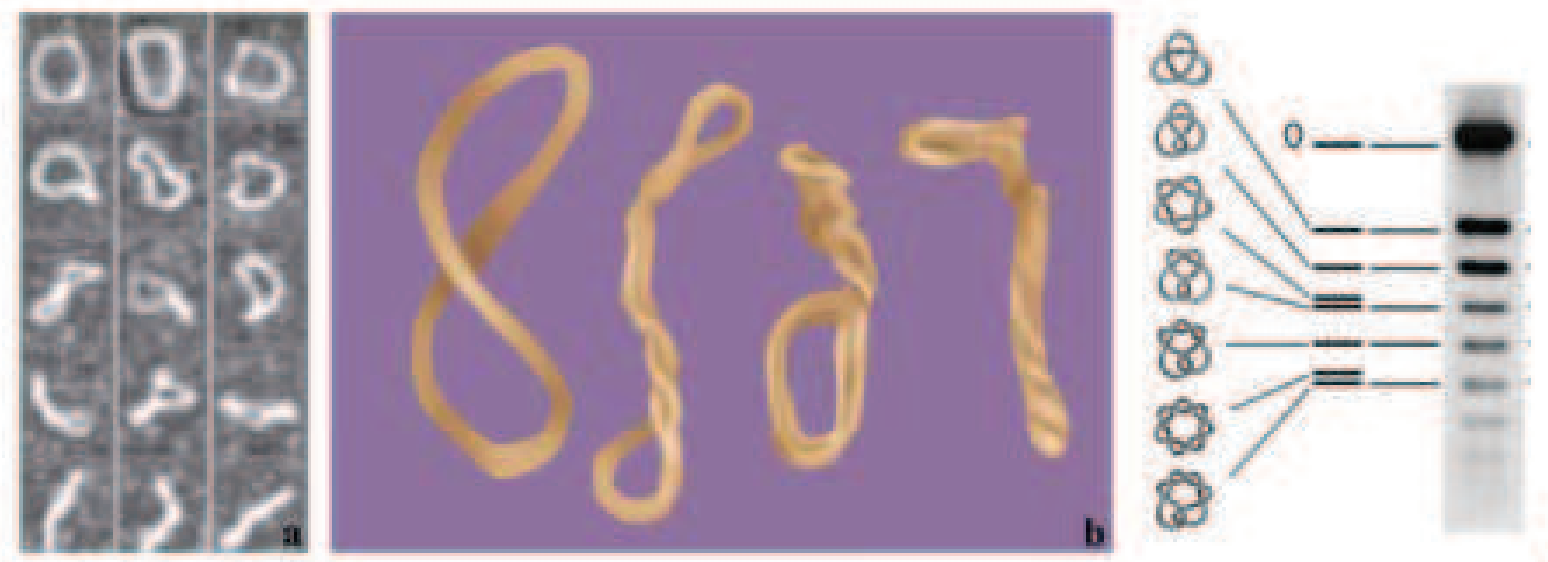

Figure 7: (a) DNA supercoiling has been explored and quantified using atomic force microscopy, a technique that allows for direct observation of unstained DNA molecules. Factors such as the length of DNA and concentration of divalent metal ions have an effect on the level of supercoiling. Image used with permission from [17]. (b) Rubber bands cut, twisted, then rejoined by using adhesive tape, can be used as a physical model of DNA supercoiling, in order to show trapped metastable confirmations. (c) Circular DNA with different knotted structures can be resolved by electrophoresis on an agarose gel. Various conformations migrate faster than the unknotted circular DNA (largest band on the top). Image used with permission from [18]. 


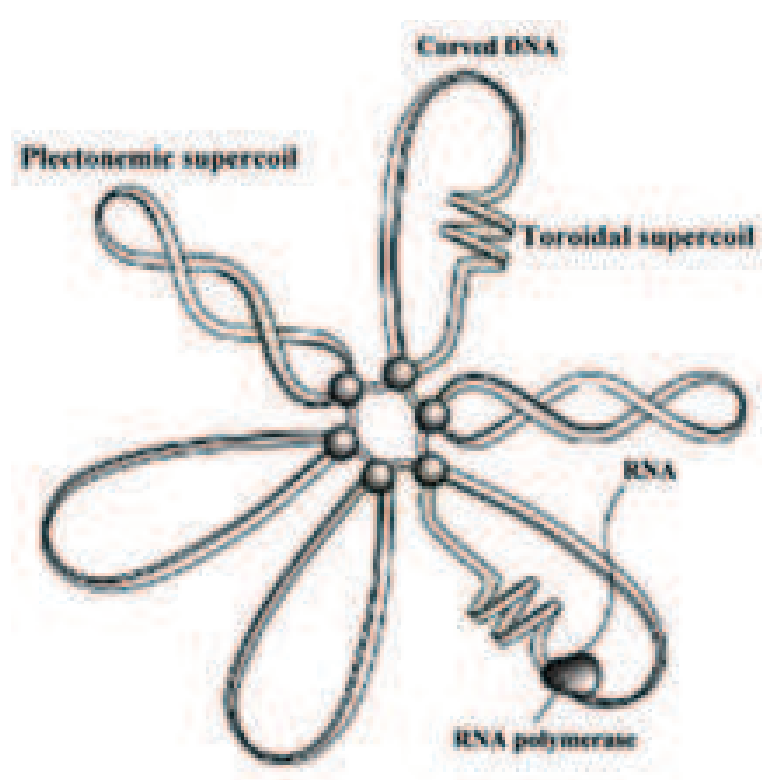

Figure 8: Plectonemic and solenoidal supercoiling of DNA. An illustration of DNA supercoiling domains in the E. coli chromosome. This is a cartoon of the chromosome; in real life there are perhaps as many as 400 different domains. Plectonemic (unrestrained) and toroidal (restrained, for example by wrapping around a protein) supercoiling is indicated. Curved DNA tends to be localized at the tips of supercoils. Adapted with permission from [19].

nodes formed by crossing of two different strands, we can determine the linking number simply by adding all crossing numbers and dividing the sum by 2 [14].

Upon application of this convention to a linking number analysis of circular DNA, one can see that the linking number is conserved even when the geometrical properties of the molecules change (Figure 11). A change in twist will result in a change in writhe, and vice versa, but the linking number does not change, unless the topological integrity of a molecule is perturbed by a topoisomerase or another enzyme which nicks or cuts the DNA thereby allowing the strands to rotate around each other. Students can explore this idea using two intertwined circular strips of Velcro-joined ribbons. By increasing the writhe, we can decrease the twist, and vice versa, but the linking number remains constant. White [20] introduced this relationship, thusly:

$$
\mathrm{L}=\mathrm{T}+\mathrm{W} .
$$

Thus, the linking number $\mathrm{L}$ has integer value and is a topological invariant while the geometric properties twisting $\mathrm{T}$ and writhing $\mathrm{W}$ are continuously deformable (see Figure 11).

\section{Demystifying negative and positive supercoiling}

DNA is negatively supercoiled inside a cell $[14,15]$. Biology students read that phrase in textbooks and often memorize it without any conceptual understanding. Negative supercoiling has a profound 

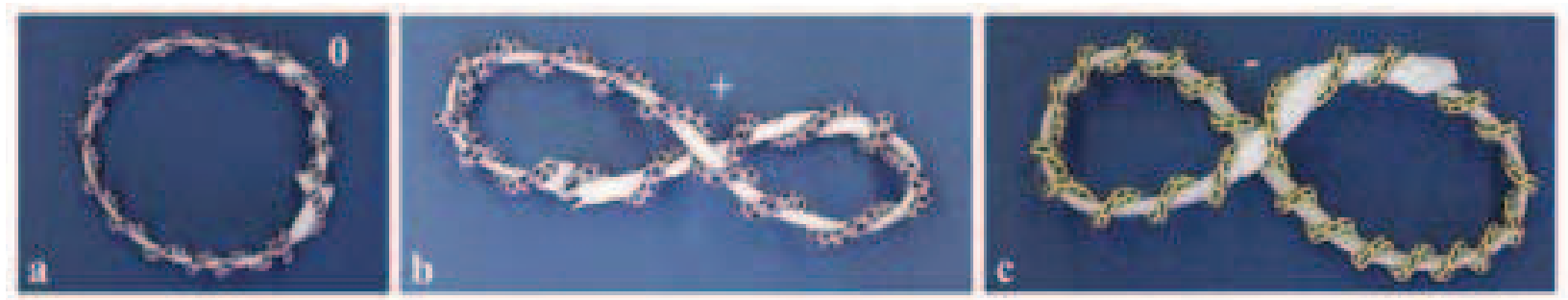

Figure 9: Simple home-made models, made of bungee cords and heavy gauge coated wire, allow students to physically manipulate models to understand conventions used to assign (a) no, (b) positive, and (c) negative crossing numbers to each intersection of DNA molecule with itself. The crossing convention is further described in Figure 10.
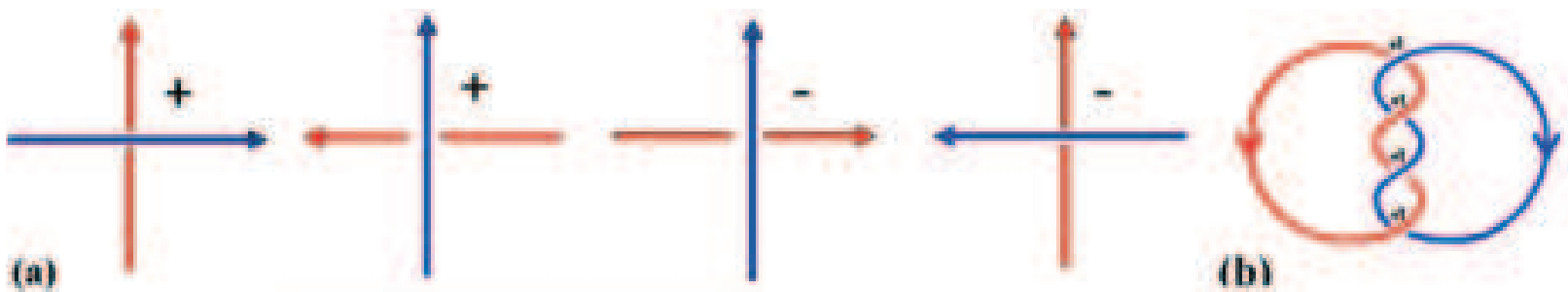

Figure 10: (a) A convention is used to assign +1 or -1 to each node, i.e. place where DNA strands cross each other, as shown above. If the over-passing segment needs to be rotated counterclockwise to overlap the under passing segment +1 is assigned $\mathrm{a}+1$, and if it needs to be rotated clockwise, it is assigned a -1. (b) An example of a curve with linking number 2. Each of the four crossings is assigned +1 , hence $\mathrm{L}=4 / 2=2$.

energetic impact on cellular processes such as replication, recombination and repair. Specifically, the underwound, negatively supercoiled DNA facilitates local denaturation between the two strands of the helix, which allows access by DNA-binding and DNA-modifying enzymes. Again, simple physical models can be used to explain what negative supercoiling means and how it is related to several biological processes.

DNA is negatively supercoiled when its linking number is lower than the linking number of relaxed DNA (Figure 12). A relaxed circular DNA will have a linking number equal to its twist, with writhe being zero. This simply means that the two strands wrap around each other with the standard helical repeat frequency (normally 10.5 bp per turn), and the double helix does not cross itself at all. If we nick or cut the relaxed circular DNA, its linking number will not change.

A negatively supercoiled DNA can be thought of as being underwound, meaning that it has fewer helical turns compared to a relaxed DNA. Thus, the linking number of DNA is lower than that of the lowest energy state. This realization brings a whole set of questions that can be discussed in classroom. What is the most thermodynamically favorable state a DNA molecule can adopt? How does negative supercoiling introduce strain into a DNA structure? What are some biological consequences of negative supercoiling? These questions can be raised in classroom discussion of the topic, and students can follow up by locating and reading relevant research articles. The 

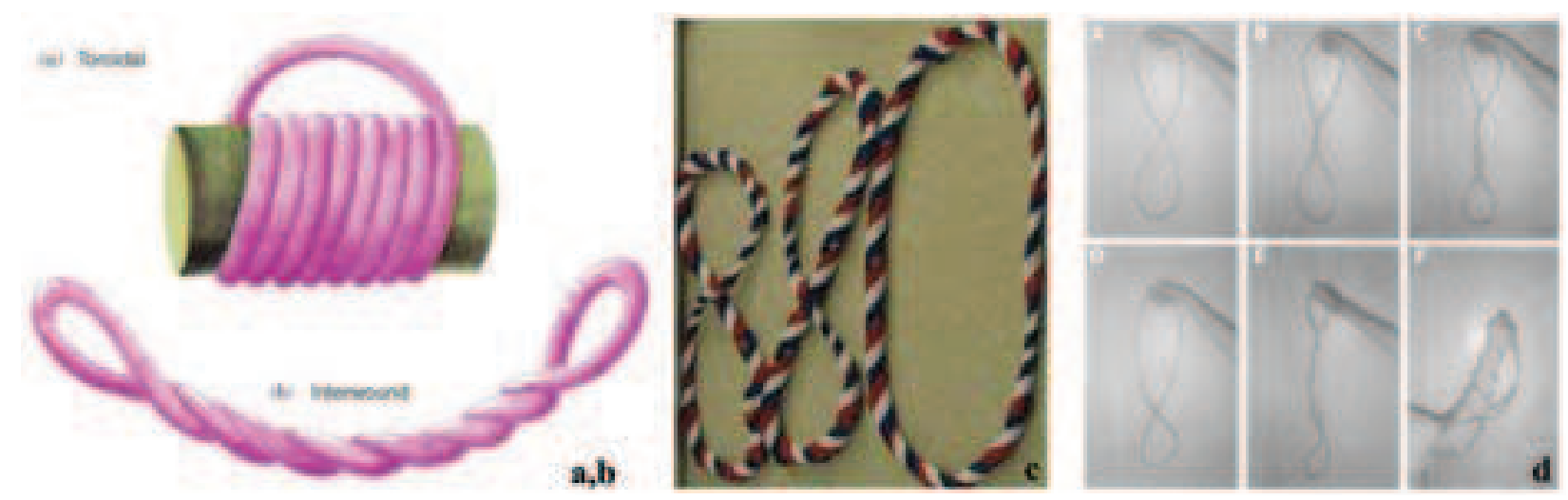

Figure 11: (a \& b) This model can be used to explore the relationship between $\mathrm{T}$ and $\mathrm{W}$. The (a) toroidal model has 8 smooth writhes while the (b) interwound model has 8 twists (Image from http://www.siumed.edu/ bbartholomew/images/chapter29/F29-21.jpg) Students can easily manipulate one geometry to the other without cutting; i.e., without changing the topology of the molecule. (c) Multicolor ribbons can be used to explore introduction of positive and negative supercoiling, as demonstrated by Milla[8]. Specifically, students may construct the three following scenarios: 1) Relaxed model: $\mathrm{W}=0, \mathrm{~L}=\mathrm{T}=22$; 2) Negatively supercoiled model: $\mathrm{L}=\mathrm{T}+\mathrm{W} .22=23$ 1; and 3) Positively supercoiled model: $\mathrm{L}=\mathrm{T}+\mathrm{W}, 22=21+1$. (d) Eckdahl demonstrates this quite differently by assuming that tube has a fixed twist and thus the linking number and writhe are coordinated [22]: "DNA Supercoiling Model Conformations. Each model represents a molecule with $\mathrm{T}=500$. (A) One positive supercoil, $\mathrm{L}=501, \mathrm{~W}=+1$. (B) Two positive supercoils, $\mathrm{L}=502$, $\mathrm{W}=+2$. (C) Three positive supercoils, $\mathrm{L}=503, \mathrm{~W}=+3$. (D) One negative supercoil, $\mathrm{L}=499, \mathrm{~W}$ $=-1$. (E) Four negative supercoils, $\mathrm{L}=496, \mathrm{~W}=-4$. (F) Four negative toroidal turns, $\mathrm{L}=496, \mathrm{~W}=$ $-4 . "$

following review articles would be a great starting point for these explorations through playing with models such as ribbons, cords and rubber bands [23,24]. The following books can be used to help with a general introduction to the topological mathematics needed to understand supercoiling and knotting of the DNA $[25,26]$.

Linking number has a clear advantage of being directly related to an experimentally measurable property of DNA: its migration on an agarose gel. Agarose gel electrophoresis is a common technique widely used by molecular biologists to separate DNA molecules of different sizes. Molecules of the same size with different levels of compactness can also be resolved. Supercoiled DNA migrates more rapidly than a relaxed DNA molecule of the same size (Figure 7c). More specifically, the linking difference, $\Delta \mathrm{L}$, allows us to experimentally study DNA topology. The linking difference $\Delta \mathrm{L}$ is defined as $\Delta \mathrm{L}=\mathrm{L}-\mathrm{L}_{0}$, where $\mathrm{L}_{0}$ is the linking number of relaxed DNA. One and two-dimensional gel electrophoresis techniques have allowed scientists to dissect complex mechanisms by directly observing topological conformations of DNA [6].

Despite the fact that the DNA is "underwound" in terms of its linking number, we know that the DNA is packed efficiently inside the cell. In fact, any non-zero $\Delta \mathrm{L}$ introduces a strain into DNA, thereby causing the DNA to adopt highly supercoiled, compact structures [27]. These structures 


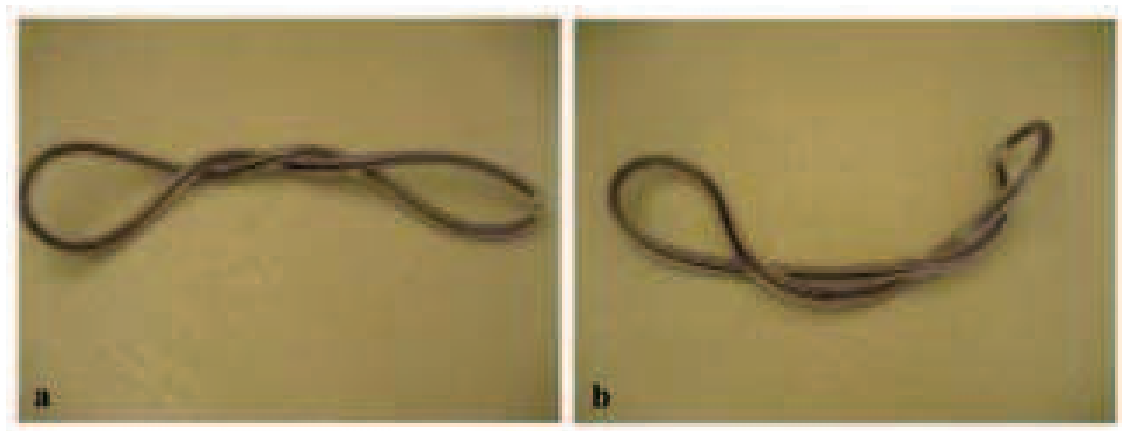

Figure 12: Positive and negative supercoiling can be modeled using a piece of Tygon tubing. By twisting one end of the tubing three times counterclockwise or clockwise, while holding the other end of the tubing steady, and then joining the ends, we can generate supercoiling. This results in positive (a) and negative (b) supercoiling. These two arrangements are mirror images of each other, however, DNA itself has chirality as a right-handed helix, so the two arrangements are radically different.

have been observed by electron microscopy using in vivo experiments. Students can model supercoiled structures using strings and ribbons, to experiment with how different levels of supercoiling affect shapes and compactness of the resulting structures. Understanding these structures helps students gain a deeper appreciation for complexity of the structural organization of DNA.

\section{Mathematical models help us understand enzyme mecha- nisms: Knots and catenanes}

How do topoisomerases change the topology of DNA? To better understand the action of topoisomerases on supercoiled DNA, we need to introduce two more topological concepts: knots and catenanes (Figure 13). A simple definition of a knot is a self-tangled circle, whereas a catenane is a set of at least two mutually intertwined circles. To detect changes in DNA toplogy caused by topoisomerases, we have to compare the structure of knots and catenanes and after processing by topoisomerases.

Although the mathematics of classifying and comparing of various knots and catenanes might not be familiar to introductory college mathematics and biology students, some of its main concepts can be easily demonstrated with strings, ribbons or tubing. One of the methods for analyzing changes in topology during biochemical processes such as replication, recombination or repair is linkage analysis. Knots and canenanes can be projected onto a two-dimensional plane (i.e., flattened), and each node (where segments cross) can be assigned a positive (+) or negative (-) sign, according to the convention shown in Figure 9. Experimentally, knots and catenanes have been successfully demonstrated and identified in DNA molecules using a combination of electron microscopy and two-dimensional gel electrophoresis [32, 33].

The crucial role of topoisomerases has been inferred from their high degree of conserva- 


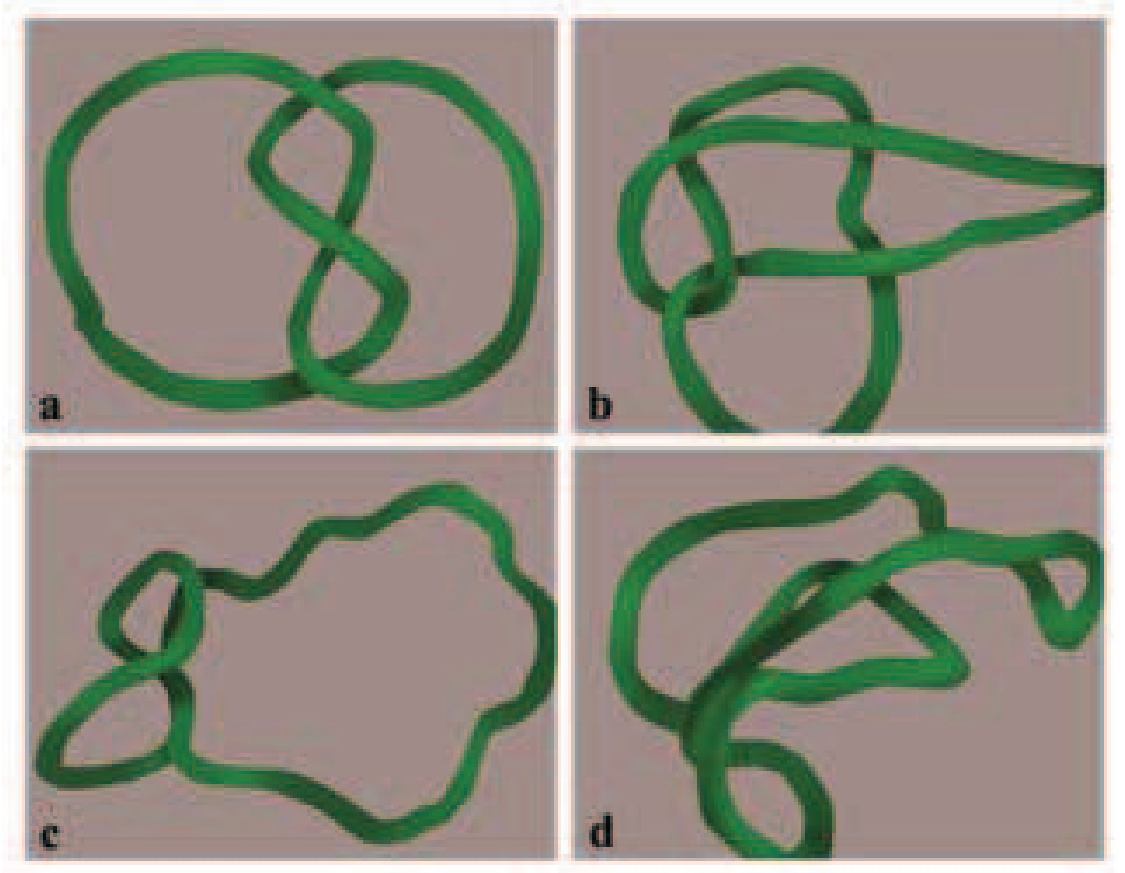

Figure 13: Although the four knots shown above appear different, they are topologically equivalent. Students can use ribbons or pipe cleaners to show that each knot can be continuously deformed into another without having to cut the ribbon. Determining whether two knots are topologically the same or different is a problem central to mathematicians. One knot is topologically equivalent to another if the two can be changed into each other without cutting. Showing that the two knots are the same can be complicated. For example, it turns out that all four knots shown in Figure 13 are actually topologically equivalent. Mathematicians have developed several tools for classifying knots, including the Alexander, Jones, Kaufmann and Conway polynomials [28]. Two knots are identical if one can be transformed into another through a series of Reidemeister moves, including twisting and untwisting, moving loops over another, and moving strings over and under crossings. The mathematics of knot classification is reviewed in [29]. Two software packages, KnotPlot (http://www.knotplot.com/) and a specialized DNA-oriented application TOPO-ICE-X can be used to plot and analyze knots $[30,31]$.

tion across all three domains of life (Figure 14a). Several important classes of drugs, including quinolone antibiotics and cancer therapy agents, such as doxorubicin, are topoisomeras inhibitors [34]. Molecular biologists have determined the basic mechanisms of several topoisomerases through clever experimental setups involving separation of DNA molecules based on their variable migration rates on gel electrophoresis caused by changes in DNA topology. Crystal structures of several different topoisomerases shed additional light on diverse ways in which these enzymes catalyze cleaving, manipulation and resealing of cut DNA strands [35]. In order to quantify and interpret the effects of various topoisomerases on the DNA, biologists rely on a combination of experimental techniques, theoretical mathematical models, and computational visualization. 


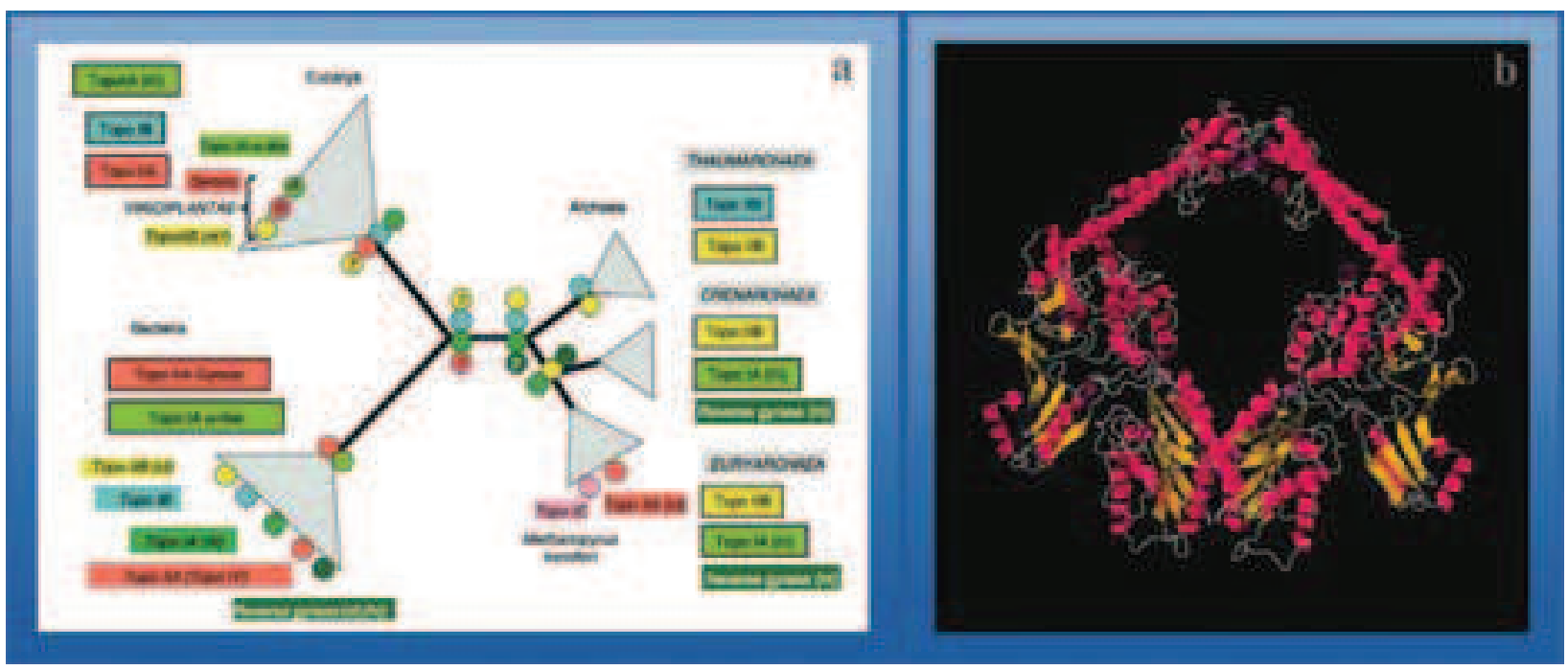

Figure 14: (a) Phylogeny of topoisomerases. DNA topoisomerases are distributed across all domains of life and highly conserved. Image used with permission from [36]. (b) Crystal structure of DNA topoisomerase II (PDB code 1BGW) shows that the enzyme has a large central hole. The two sets of "jaws" allow the enzyme to bind two DNA duplexes at a time, allowing for movement and resealing of two cut strands of DNA [37]. Mathematicians predicted ahead of experimental observations that these enzymes would have a hole $\geq 20 \AA$ in order to accomodate passing a DNA double helix through another while binding the two ends of the broken double strands and then resealing. Students can take the PDB file into a standard protein visualization software package to measure the diameter of this hole.

Topoisomerases change the DNA topology by changing the linking number of DNA molecules. A change in the linking number as a result of enzymatic action implies that at least one of the DNA strands was broken and resealed (religated), i.e., the DNA topology has changed. We now know that all topoisomerases do exactly that. Type I topoisomerases break one strand of DNA and reseal it after rotating the strand around the other one, whereas Type II topoisomerases introduce a double-stranded break, allowing the strands to rotate and then resealing the break [38, 39, 40] (Figure 14b). Type I topoisomerases change the linking number by $+/-1$, whereas class II enzymes change the linking number by $+/-2$. The action of type II topoisomerase can be demonstrated using a two-colored ribbon (Figure 15a,b,c). This model is similar to one in which one of the "strands" is sewed together and the other strand can be cut and resealed with Velcro. Two interwould tubes could be used to model Type I topoisomerases (Figure 15d,e).

We recommend that you give students a banded ribbon such as illustrated in Figure 15 but with either two twists or two writhes in it (it should look like a triple " 8 " when laid out; i.e., with three bounded regions instead of 2). If one student acts as the topoisomerae by rigidly holding their hands out with a clear hole between their arms and grasps the ribbon with one hand on either side of the Velcro joint, then they can behave like a Type II topoisomerase and simply cut or re-join the two ends of the ribbon without ever having let go of either end. If other students are asked to 


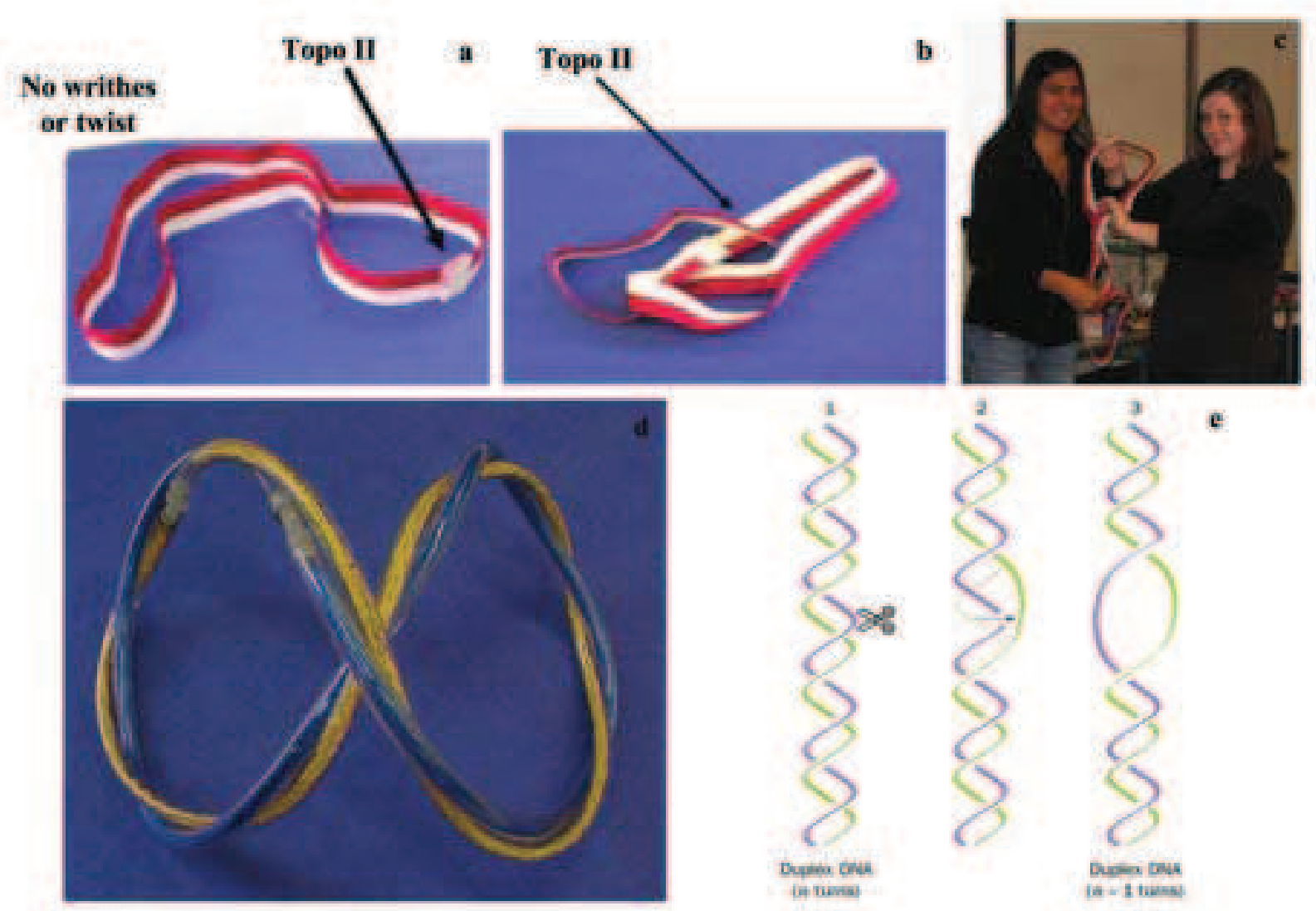

Figure 15: (a) A ribbon model with two colors to indicate the two antiparallel Watson and Crick strands of a DNA can be used to model the action of class II topoisomearse. Breaking the Velcro connection between the two strands is analogous to a double stranded break in the DNA. (b) After introducing a twist, the ribbon is resealed so that the red and white areas are continuous. This changes the linking number of the DNA modeled by ribbons. (c) Topoisomearse II model with a DNA with linking number $=2$ isillustrated by Beloit College students Raksha Ashlaysha and Melissa Kattke. (d) Tygon tubing filled with blue and yellow plastic coated copper wires and coupling connectors are nice for illustrating Topoisomerase I reactions with DNA. (e) Relaxation of a double stranded DNA by catalyzed Topoisomerase I reaction [Source: http://www.siumed.edu/ bbartholomew/images/chapter29/F29-25b.jpg].

manipulate the DNA so as to remove the twists or writhes, you can usually enjoy several minutes of fun before they realize that by simply moving the double stranded molecule through the open hinged regions and then rejoining the loose ends they can either introduce or remove twists or writhes. 


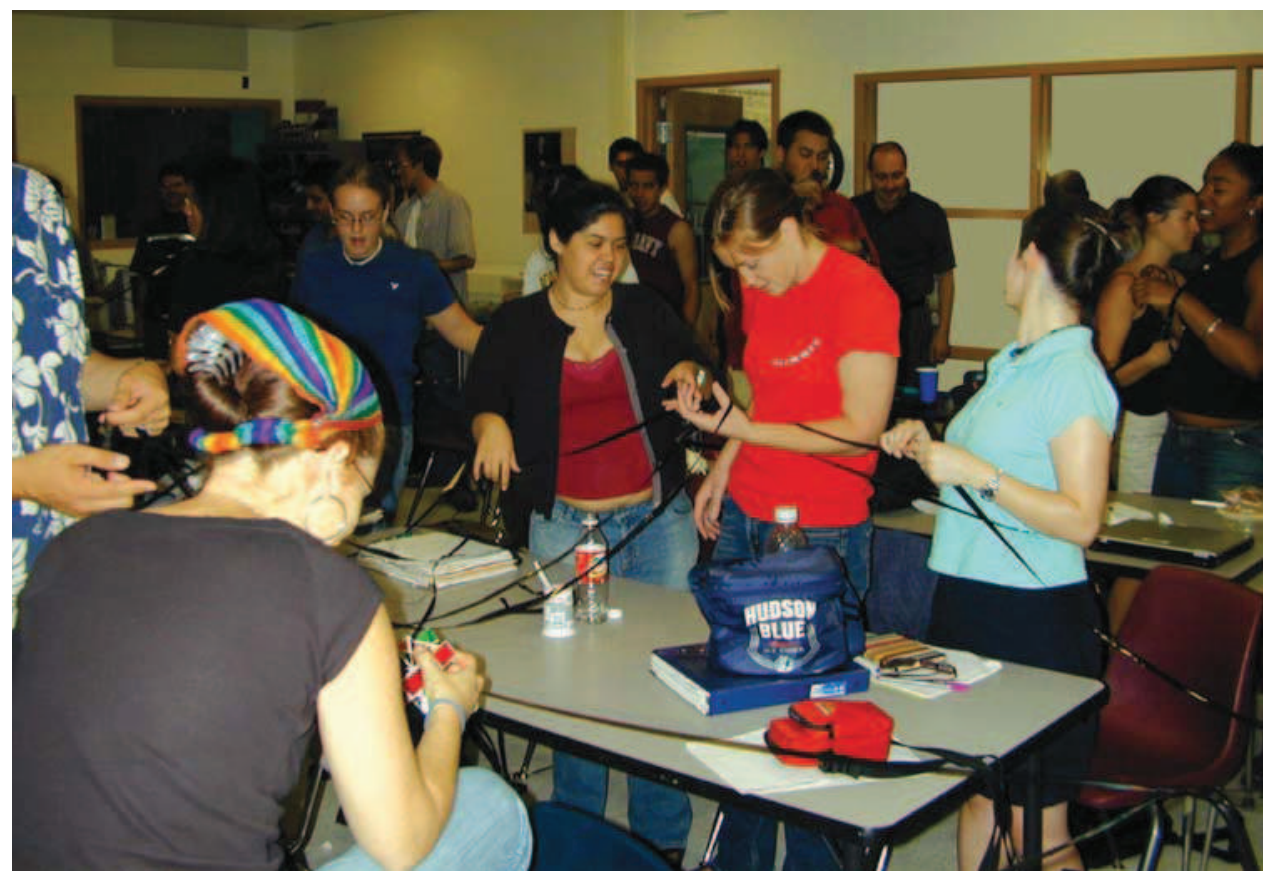

Figure 16: Mathematical and Theoretical Biology Institute (MTBI) at Los Alamos National Labs led by Professor Carlos Castillo-Chavez of Cornell University (he and the program have since moved to Arizona State University); despite having gone through demonstrations of packing, the physical process of completely emptying the cassette and seeing the total length of tape at once still managed to amaze them. [http://mtbi.asu.edu]

\section{Untangling the chromosome: What happens to strands dur- ing replication?}

Supercoiled molecules are more compact than relaxed molecules; hence supercoiling is at least a part of the explanation of the amazing efficiency with which DNA is packaged inside a cell. The genome of the non-pathogenic bacterium Escherichia coli is about 4.6 megabases [41]. Assuming the distance of $0.33 \mathrm{~nm}$ between successive base pairs, we can calculate its total length to be approximately $1.5 \mathrm{~mm}$. Given that the diameter of the whole $E$. coli cell is $2 \mu \mathrm{ms}$, it is obvious that the circular DNA genome is significantly compacted (750 fold compaction). It might be difficult to visualize this on a sub-millimeter scale. To demonstrate this level of packing, students could be challenged to pack a 15 meter string so that it fits into a ball with a diameter $2 \mathrm{~cm}$ (a ping pong ball might serve as a model). Another powerful visualization of the result of efficient packing is pulling all the tape out of a video cassette (Figure 16). This classroom demonstration amazed a large group of scientists at a AAAS annual meeting's symposium "Science Is Fun" and at an ASBMB annual meeting as well.

Clearly, to achieve this level of efficient packing, the string has to be extremely tangled or tightly wound up. How then is the DNA unwound during replication? The answer to that question 
involves a complex set of finely-tuned enzymatic reactions. Helicases, enzymes which unwind the DNA strands at the replication fork, introduce positive supercoils ahead of the replication fork [42]. The strands can not rotate freely around each other because they are constrained by the circularity in the case of bacterial chromosomes or by attachment to histones in the case of eukaryotic DNA [43].

\section{DNA structure in a compact chromosome - How can three dimensions be filled with a one-dimensional string?}

Many textbooks reproduce a conventional description of the compaction of DNA in a chromosome as a coiled-coiled-coiled-coiled-coil (DNA double helix - wrapped around histones to form a nucleosome - nucleosomes coiled into stringy coils called polysomes -polysomes in turn coiled into loops - and - then, finally, these loops of polysomes compacted into chromosomes) ( Figure 17). Nicholl and Nicholl had students model these structures with styrofoam balls and wire for nucleosomes and polysomes (Figure 18)[44].

However, recent work modeling the compaction of eukaryotic DNA at the megabase scale has demonstrated even more compaction than the solenoid levels typically depicted in textbook images [45]. In 1988, Grosberg and colleagues predicted that DNA would fold into a fractal compact structure that they referred to as "globules" [46]. Lieberman-Aiden concluded their experimental study of proximity maps of the whole genome by inferring the extremely compact chromatin conformation is consistent with fractal globule model (Figure 19)[45]. A model of a fractal globule is a compact, knot-free confirmation. A fractal globule model resembles a Peano curve, a continuous curve which fills the space densely without crossing itself. Small pieces of DNA fold into globs, and those globs fold into larger globs and so on. The researchers report that this "globule of globules of globules" is fractal, meaning it is organized in such a way that it has a self-similar pattern no matter how far you zoom in. This fractal shape is "super-dense", but has no knots (http://fractalfoundation.org/2009/11/fractal-globules-in-dna/). Furthermore, Buenemann and Lenz have also developed a bacterial chromosomal model with a fractal compaction of a chromosome [47]. The absence of knots and entanglements could play a role in making the DNA more amenable to folding and unfolding during processes such as gene activation or repression.

In order for students to develop some sense of such compact packings, we like to use modeling Toobers ${ }^{\mathrm{TM}}$ (http://www.3dmoleculardesigns.com/toobers.php ) to create modified Peano curves in both two and three dimensional packing schemes. This activity helps them understand how a onedimensional molecule can completely fill two- and three-dimensional spaces. In the future, we plan to have students study the work of Jason $\mathrm{Ku}$ who has built physical models of these fractal packings with origami (Figure 20). 

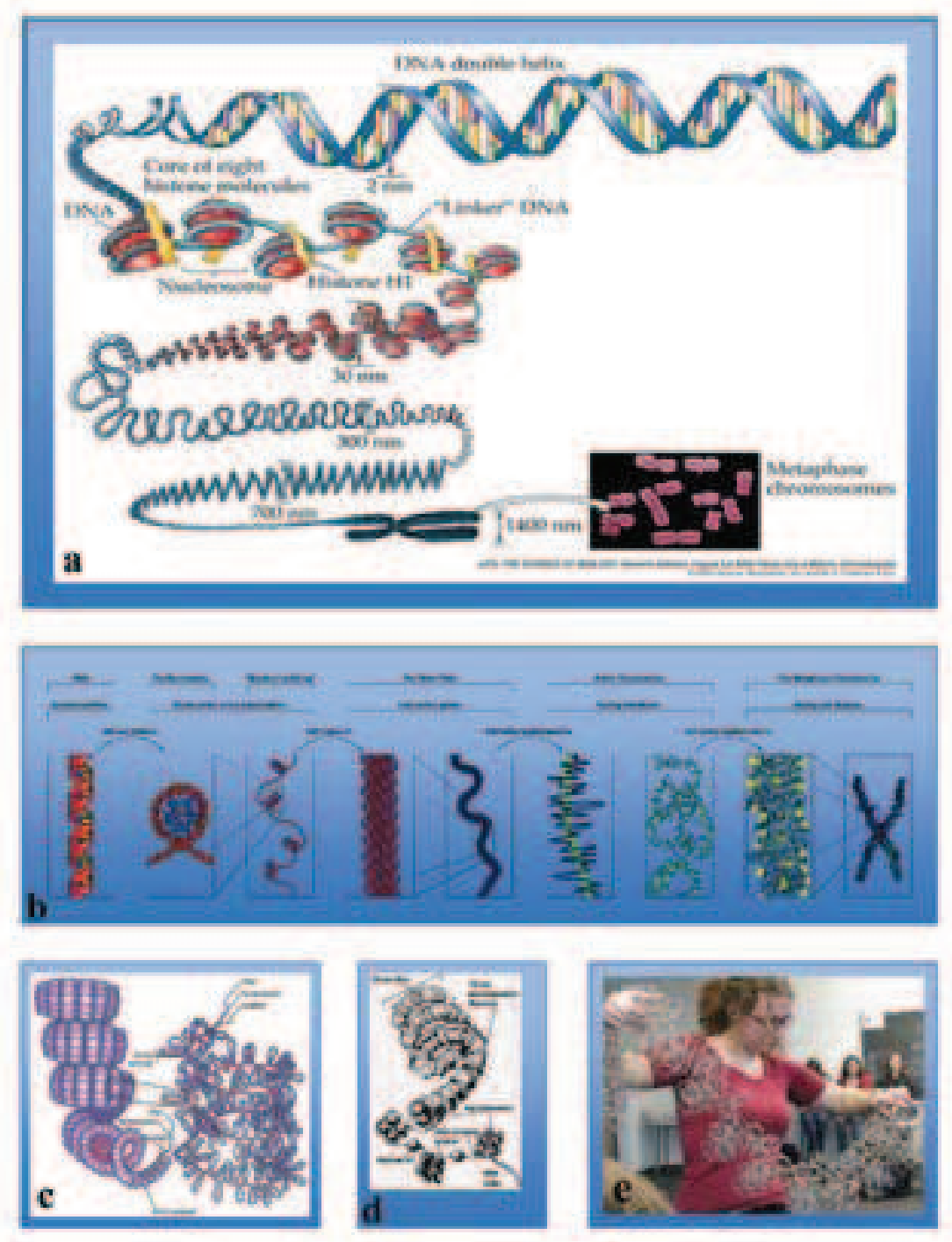

Figure 17: The compaction of DNA in a chromosome as a coiled-coiled-coiled-coiled-coil: (a) The five levels of coiling are displayed here in connected horizontal stripes. Image from Life: The Science of Biology, 7th Ed. (2004) Sinauer Associates: Sunderland, Massachussetts; and W.H. Freeman: San Francisco.; (b) The five levels of coiling are displayed here in the second level of brackets that clump the nine images below them; (c) solenoid structure of a polysome with scaffold highlighted; (d) solenoid structure of a polysome with individual octamers of 140bp of DNA wrapped around eight histone proteins. Images b, c, and $\mathrm{d}$ are available via a Creative Commons license at WikiBooks. (e) Beloit student Emerson Fuller with a model of coiled polysomes by Space Weaver artist Dennis Dreher. 

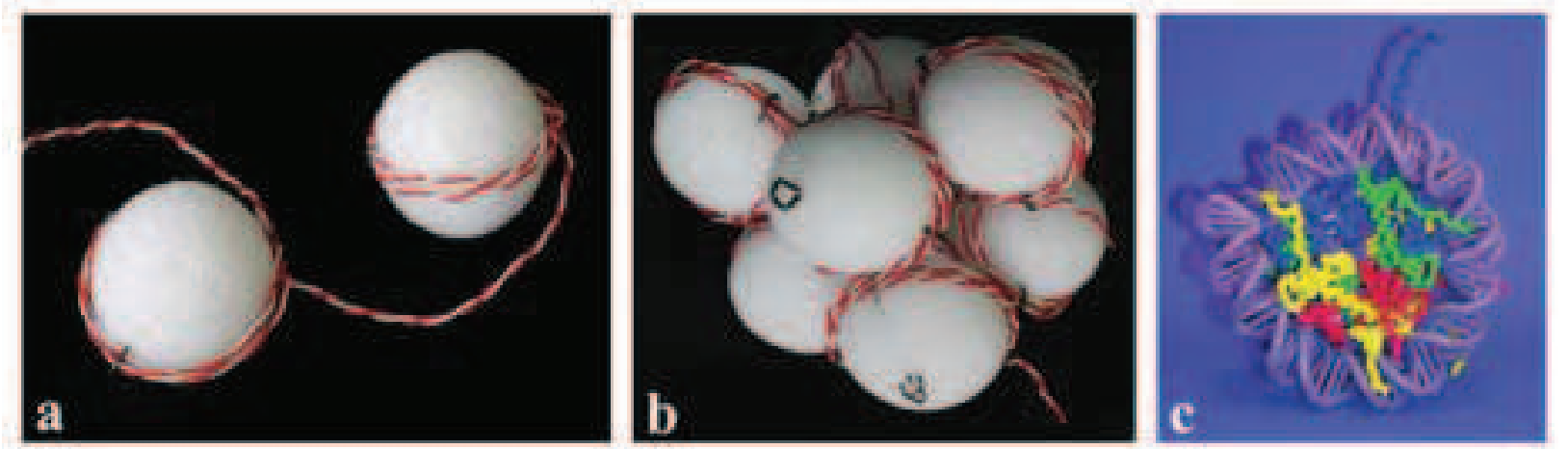

Figure 18: Styrofoam and braided wire model of DNA wound around histones to make a nucleosome (a) and several nucleosomes bundled into a section of a polysome (b). Original model was developed by Nicholl and Nicholl [44]. (c) Nucleosome model produced via three dimensional printing (positive stereolithography) by students at the Milwaukee School of Engineering's Center for Biomolecular Modeling (courtesy Director Tim Herman). The Center distributes a traveling exhibit/lending library of 3D models as well as producing models in educational workshops and by commission [10].

\section{Catenanes: Another possibility for packing DNA}

Protozoan parasites, responsible for a number of major human infectious diseases such as trypanosomiasis and leishmaniasis, that affect millions of people primarily in Africa and South America, have an organelle called a kinetoplastid which contain numerous minicircles [48]. These minicircles are interlocked (Figure 21a). Following the suggestion of Sylvia Spengler at University of California Berkeley, we have found that these are interestingly modeled with chain mail (Figure 21b). Many of our students interested in science fiction and fantasy games have been particularly intrigued with the notion that chainmail was invented by evolution over millions of years ago [50]. In one species, Leismania tarentole, the number of minicircles in an organelle may range from five to twenty thousand copies.

\section{Beyond strings and tubing - computational models and sim- ulations of DNA structure}

Despite of our general understanding of DNA structure and its regulation by enzymes such as helicases, gyrases, and topoisomerases, many interesting questions remain in the field. We are only beginning to appreciate the role of DNA topology in mechanisms of regulation of gene expression [51].

Computational models, such as Monte Carlo simulations, have provided additional insight to models of DNA supercoiling. Simulations have shown that supercoiling contributes to bringing together distant sequence sites; such supercoiling is involved in recombination and transcriptional 


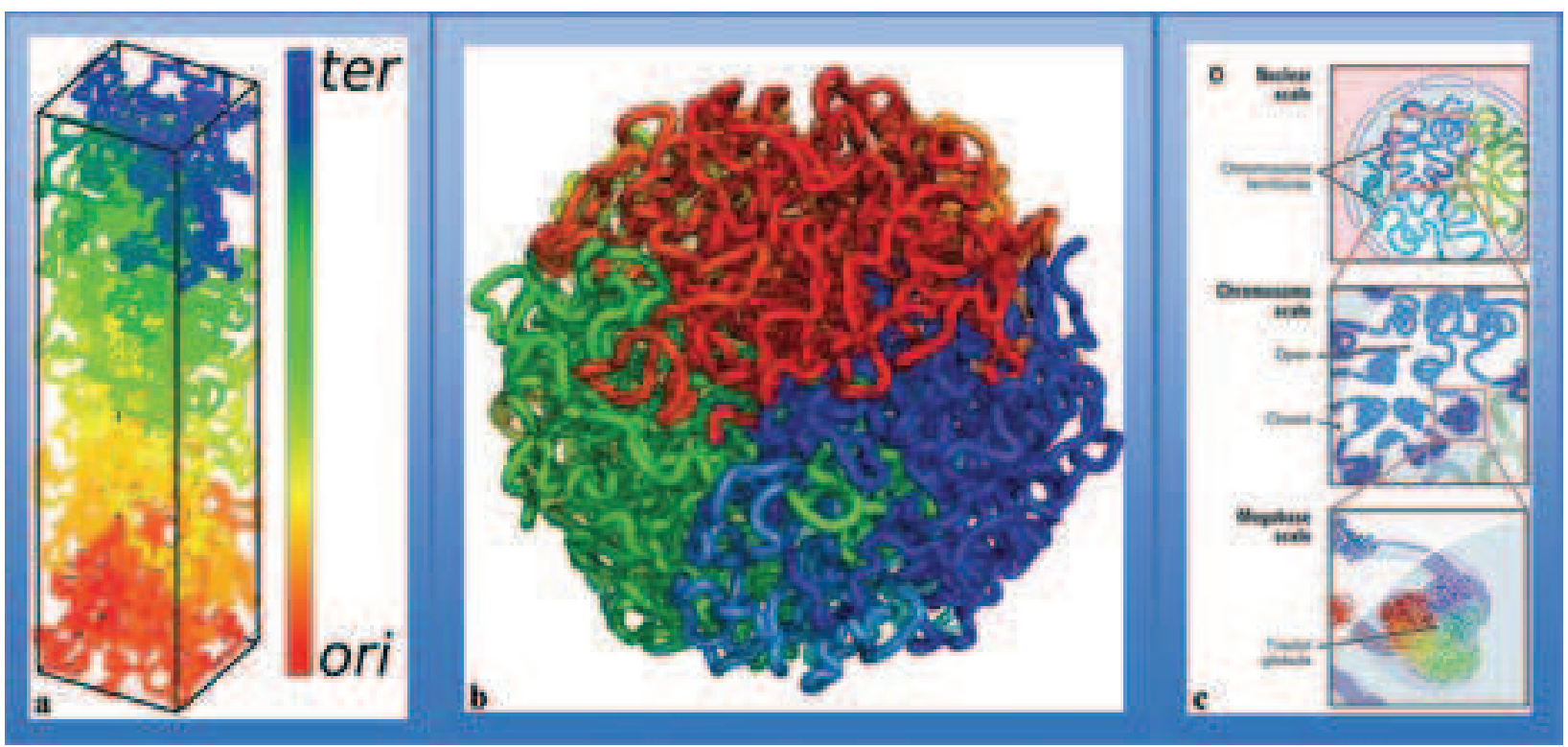

Figure 19: (a) "Typical DNA configuration of an individual Caulobacter crescentus cell belonging to a population that has an average DNA configuration showing the linear correlation between position of genes on the chromosome and position in the cell."[47]. (b) Fractal globule [image by Leonid A. Mirny and Maxim Imakaev appeared in Susannah F. Locke, "How Your Body Packs Two Meters of DNA Into a Six-Micron Cell Nucleus," http://www.popsci.com/science/article/200910/how-does-your-body-cram-all-sgrahams-dna] Quote: "First, the researchers found that a cell's DNA is organized in two compartments: an 'off' area and an 'on' area. Genes that aren't being used are tightly packed into the globule that acts like a filing cabinet, storing lots of info for later use. Genes that are currently being used are all together in a second area, where enzymes can access their sequences. Genes snake back and forth between the compartments as they're turned on and off. But how does that happen without everything becoming a tangled mess? Enter the fractal globule. This paper presents the first time this highly organized structure of strands has ever been observed anywhere. It keeps genes that are near each other on a strand also near each other in the ball, so that any point can be pulled in and out without knotting. 'It makes it easy to pull a location out, read it, and then crumble it back and push it back where you took it from,' lead author Erez Lieberman-Aiden, of Harvard University, told Popsci.com." (c) Position of a fractal globule in a eukaryotic chromosome [45].

activation [52].

Another question that remains to be answered is how topoisomerases distinguish entangled substrates they act upon from other DNA sequences. In other words, how can topoisomerases "sense" the overall entanglement of the DNA molecule? Clearly, the thermodynamics of various conformations must influence the enzymatic activity of topoisomerases, but exactly how is yet to be determined. Interestingly, it has been shown that type-II isomerases catalyze the conversion of DNA molecules to an ensemble of confirmations containing less than the equilibrium value of knotted and linked molecules [53]. This is in stark contrast to other enzymes which catalyze 


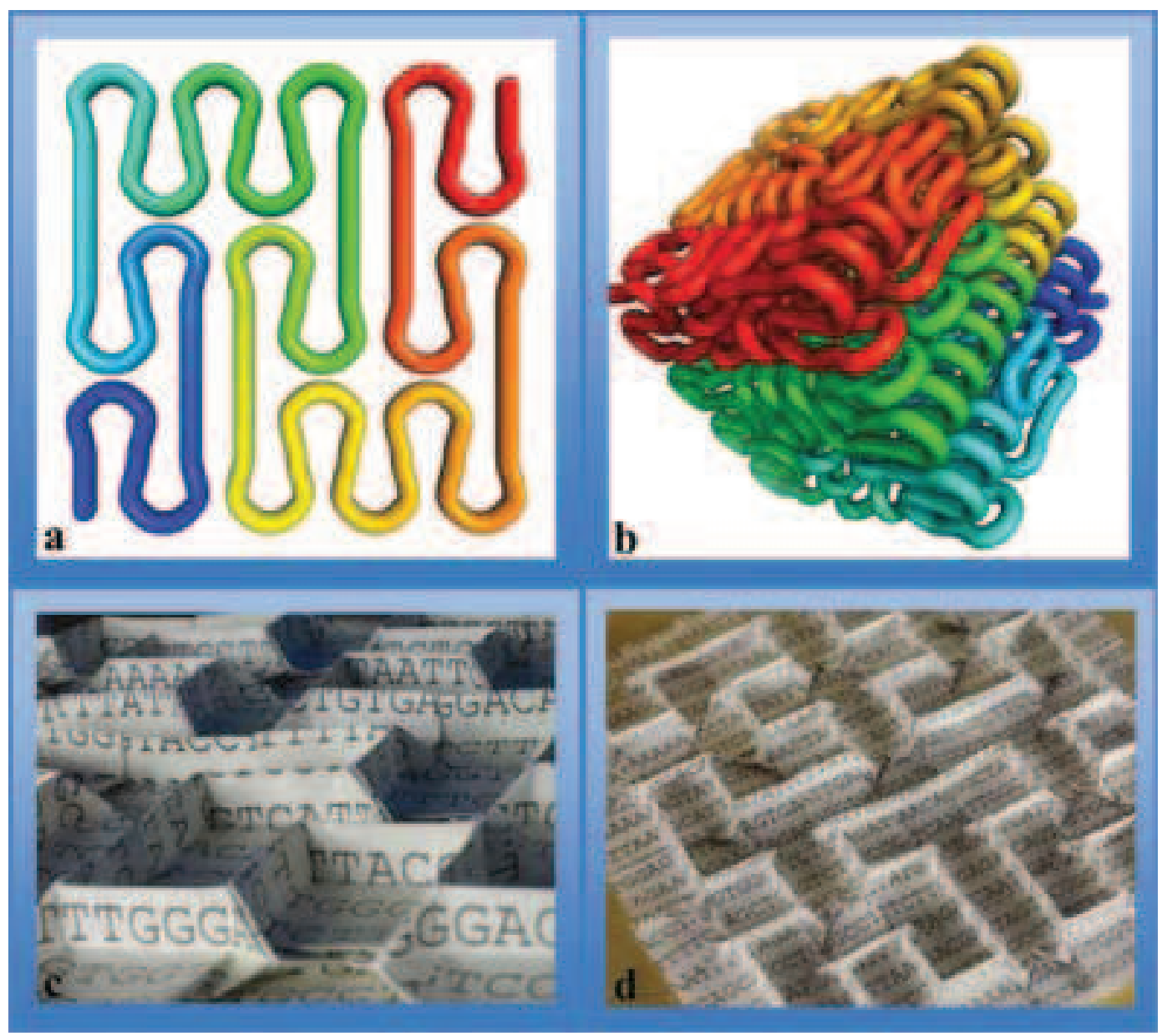

Figure 20: (a) Two dimensional space filling of a Hilbert curve, a variant of the space-filling curves discovered by Giuseppe Peano in 1890 , modeled with a Toober ${ }^{\mathrm{TM}}$. Image used with permission from [48]. (b) Three dimensional space filling of a Hilbert curve modeled with a Toober ${ }^{\mathrm{TM}}$. Hilbert curve us a one-dimensional fractal trajectory that densely fills higher-dimensional space without crossing itself. First described by David Hilbert in 1891, the Hilbert curve is a one-dimensional fractal trajectory that densely fills higher-dimensional space without crossing itself. (c) and (d) Jason Ku's origami model of a DNA fractal globule model of human chromosome 14. [Designed and folded by Jason Ku. Photo by Erik Demaine. Thanks to Brian Chan, Erik Demaine, Martin Demaine, and Erez Lieberman-Aiden.]

reactions until the equilibrium distribution of reactants and products is achieved. Monte Carlo simulations of topoisomerase action on a worm-like chain model of DNA show that this might be achieved through a bend in the DNA [54]. Despite this insight, mechanistic details and energetic consequences of simplification of DNA topology by type-II topoisomerases are yet to be explored. 


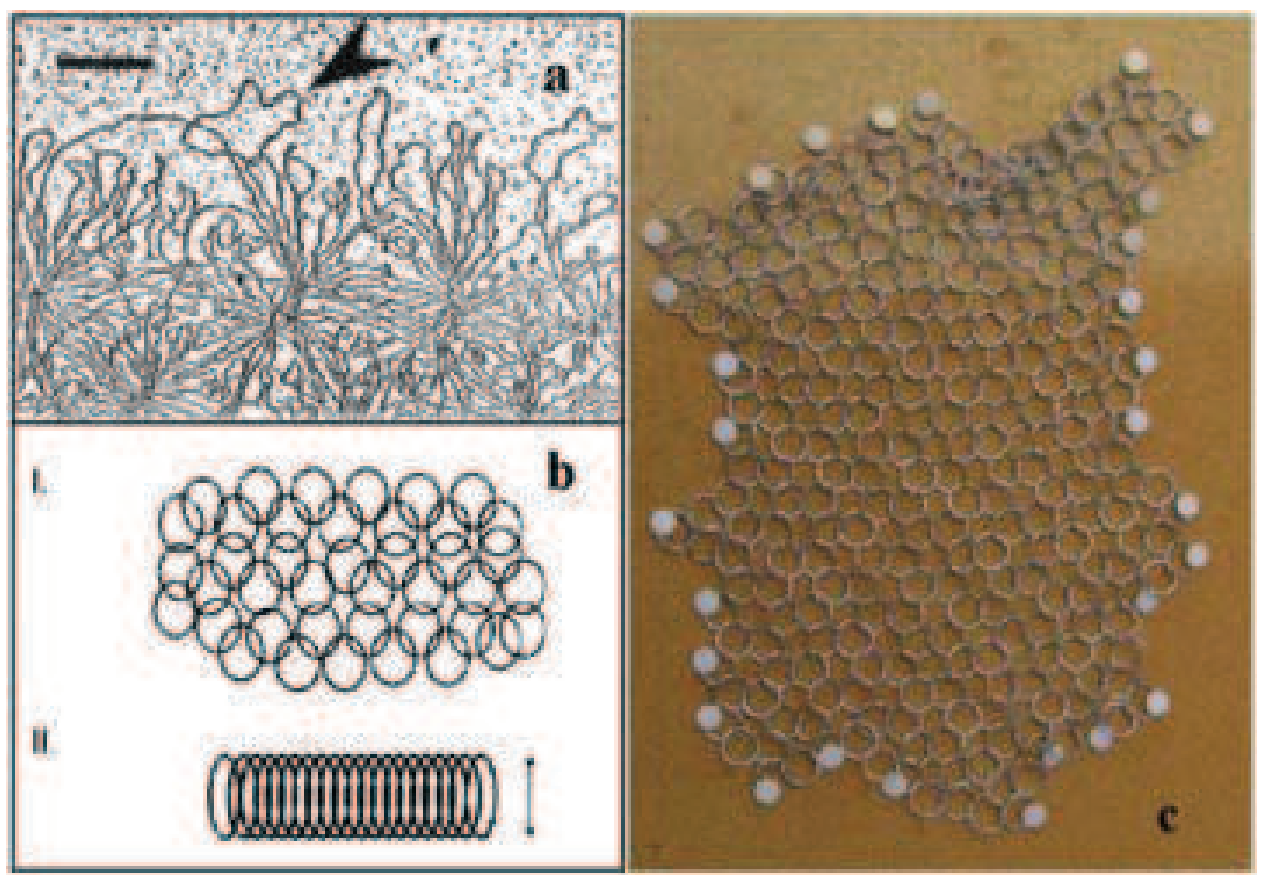

Figure 21: (a) Kinetoplastid minicircles: electron micrograph and graphical display of their interlocking and compaction. Except for minicirciles on the outer periphery, each minicircle is catenated to three other minicircles via a single interlock. [http://ec.asm.org/content/vol11/issue4/images/large/ek0420038001/jpeg] [48] (b) Kevin Braun, a chemist at Beloit College, constructed this chain mail model of Kinetoplastid minicircles. [49].

\section{Conclusion}

The complex task of maintaining and regulating multiple levels of DNA structure, such as supercoiling, knotting, and concatenation between DNA strands, is accomplished through a highly coordinated action of DNA-modifying enzymes. These enzymes play important roles in all living cells and organisms, and must be finely tuned to respond to slight changes in DNA topology. The field of DNA topology regulation is an example of a very successful interplay of mathematical modeling, computational vizualization, and experimental biology. Currently, new developments in singlemolecule experiments and computational modeling are helping us develop a theoretical framework for understanding both local and global levels of regulation of DNA topology [54, 55, 56]. By providing undergraduate students with opportunities to gain competencies in both mathematics and biology, we prepare them for success in increasingly more interdisciplinary research endeavors. After mastering a few basic concepts of DNA topology through hand-on explorations with simple toys, undergraduate students will be able to follow these exciting new developments, and develop a deeper appreciation for the importance of mathematical modeling in biological research.

Furthermore, interactive topological modeling such as illustrated herein confers some additional general mathematical educational benefits as well in that it usually induces some metacognitive reflections that generally could be restated as: Mathematics isn't just about numbers and equa- 
tions, but idea processing as well. Mathematics isn't just about getting answers, but frequently is most helpful when we are challenged by an unexpected or counterintuitive idea. Complex phenomena can be understood more deeply with simple mathematical abstractions. In that most students in biology have never had a course in topology and if they have had any contact at all, it has usually been limited to one or two students in a whole class that have explored a Möbius strip, this excursion in topological ideas helps them appreciate that there are very different kinds of mathematics than just calculus and statistics that are appropriate for reasoning about biological problems and, furthermore, that mathematical analysis may be as important as experiments in terms of understanding what is going on. We usually finish an hour by discussing the possibilities that mutually biology may stimulate the development of new mathematics and that mathematics may serve as a lens for designing new experiments and theoretical abstraction in biology.

\section{Acknowledgements}

We thank Nicholas R. Cozzarelli and De Witt Sumners for providing S.R. with an opportunity to participate in research and workshops related to DNA topology as an undergraduate student, Philip D. Straffin [57] for teaching an inspiring course in topology, and Giulietta Spudich and Ted Gries for critical reviews of the manuscript. JRJ is especially grateful to artist Dennis Dreher for his insightful kinesthetic knowledge of three dimensional topology as well as for numerous models that he has constructed for JRJs teaching collection. We are grateful to all the researchers in the field of DNA topology who share their images and resources with educators, and allowed us to include some of their images in this publication (especially the Zechiedrich group at the Baylor College of Medicine, Dave Ussery at the Technical University of Denmark, Julius Lukes, at the Instutite of Parasitology, Czech Republic, and Patrick Forterre at the Institut de Gntique et Microbiologie, Universit Paris-Sud, France).

\section{References}

[1] S. Elrod, W. Stansfield. Schaum's Outline of Genetics, Fifth Edition. McGraw-Hill, New York, 2010.

[2] R. Brooker, E. Widmaier, L. Graham, P. Stiling. Biology, Second Edition. McGraw-Hill, New York, 2010.

[3] N. Campbell, N. A. Reece, J. B. Jackson, R. B. Cain, M. L. Urry, L. A. Wasserman, S. A. Minorsky. Biology, Ninth Edition. Benjamin Cummings, San Diego, 2011.

[4] P. Karp. Cell and Molecular Biology: Concepts and Experiments, Fifth Edition. Wiley, New York, 2007.

[5] W. H. Elliott, D. C. Elliott. Biochemistry and Molecular Biology, Fourth Edition. Oxford University Press, Oxford, U.K., 2009. 
[6] D. W. Sumners, C. Ernst, S. J. Spengler, N. R. Cozzarelli. Analysis of the mechanism of DNA recombination using tangles. Q. Rev. Biophys. 28 (1995), 253-313.

[7] N. R. Cozzarelli, J. C. Wang. DNA Topology and Its Biological Effects. Cold Spring Harbor Monograph Series 20, 1990.

[8] G. Balliano, P. Milla. Topology of DNA: When manipulation supports the lack of "spacefilling” imagination. Biochemical Education, 25 (1997), 209-210.

[9] J. R. Jungck, H. Gaff, A. E. Weisstein. Mathematical manipulative models: In defense of “Beanbag Biology”. CBE Life Sci Educ. 9 (2010), 201-211.

[10] J. R. Roberts, E. Hagedorn, P. Dillenburg, M. Patrick, T. Herman. Physical models enhance molecular three-dimensional literacy in an introductory biochemistry course*. Biochemistry and Molecular Biology Education, 33 (2005), 105-110.

[11] T. Herman, J. Morris, S. Colton, A. Batiza, M. Patrick, M. Franzen, D. S. Goodsell. Tactile teaching: exploring protein structure/function using physical models. Biochem. Mol. Biol. Educ. 34 (2006), 247254.

[12] "kitefrog”. Möbius Strip: New Discoveries. 2011 (2008).

[13] E. Babaev, Intuitive Chemical Topology Concepts (Chapter 5), in: D. Bonchev, R. Rouvray (Eds.), Chemical Topology: Introduction and Fundamentals. Gordon and Breach, 1999, pp. 167-264.

[14] A. D. Bates, A. Maxwell. DNA Topology. Oxford University Press, New York, 2005.

[15] T. C. Boles, J. H. White, N. R. Cozzarelli. Structure of plectonemically supercoiled DNA. J. Mol. Biol. 213 (1990), 931-951.

[16] C. D. Hardy, N. J. Crisona, M. D. Stone, N. R. Cozzarelli. Disentangling DNA during replication: a tale of two strands. Philos. Trans. R. Soc. Lond. B. Biol. Sci. 359 (2004), 39-47.

[17] J. M. Fogg, N. Kolmakova, I. Rees, S. Magonov, H. Hansma, J. J. Perona, E. L. Zechiedrich. Exploring writhe in supercoiled minicircle DNA. J. Phys. Condens Matter. 18 (2006), S145S159.

[18] J. Arsuaga, M. Vazquez, P. McGuirk, S. Trigueros, D. Sumners, J. Roca. DNA knots reveal a chiral organization of DNA in phage capsids. Proc. Natl. Acad. Sci. U. S. A. 102 (2005), 9165-9169.

[19] H. Willenbrock, D. W. Ussery. Chromatin architecture and gene expression in Escherichia coli. Genome Biol. 5 (2004), 252.

[20] J. H. White. Self-linking and the Gauss integral in higher dimensions. American Journal of Mathematics. 91 (1969), 693-728. 
[21] W. R. Bauer, F. H. Crick, J. H. White. Supercoiled DNA. Sci. Am. 243 (1980), 100-113.

[22] T. T. Eckdahl. Investigating DNA supercoiling. The American Biology Teacher. 61 (1999), 214-216.

[23] J. M. Fogg, D. J. Catanese, G. L. Randall, M. C. Swick, L. Zechiedrich. Differences between positively and negatively supercoiled DNA that topoisomerases may distinguish (Chapter), in Mathematics of DNA Structure, Function and Interactions The IMA Volumes in Mathematics and its Applications, 150 (2009), 73-121.

[24] G. Witz, A. Stasiak. DNA supercoiling and its role in DNA decatenation and unknotting. Nucl. Acids Res. 38 (2010), 2119-2133.

[25] M. D. F. Kamenetskii. Unraveling DNA: The most important molecule of life, John Wiley \& Sons, 1997.

[26] A. Sossinsky, G. Weiss. Knots: Mathematics with a twist. Harvard University Press, 2002.

[27] L. Postow, N. J. Crisona, B. J. Peter, C. D. Hardy, N. R. Cozzarelli. Topological challenges to DNA replication: Conformations at the fork. Proc. Natl. Acad. Sci. USA 98 (2001), 82198226.

[28] L. H. Kauffman, S. Lambropoulou. On the classification of rational tangles. Advances in Applied Mathematics. 33 (2004), 199-237.

[29] C. Adams. The Knot book: An elementary introduction to the mathematical theory of knots. W.H. Freeman, 1994.

[30] I. K. Darcy, R. G. Scharein, A. Stasiak. 3D visualization software to analyze topological outcomes of topoisomerase reactions. Nucleic Acids Res. 36 (2008), 3515-3521.

[31] P. Freyd, D. Yetter, J. Hoste, W. B. R. Lickorish, K. Millett, A. Ocneanu. A new polynomial invariant of knots and links. Bull.Amer.Math.Soc.(N.S.). 12 (1985), 239-246.

[32] J. D. Griffith, H. A. Nash. Genetic rearrangement of DNA induces knots with a unique topology: implications for the mechanism of synapsis and crossing-over. Proc. Natl. Acad. Sci. USA 82 (1985), 3124-3128.

[33] S. Trigueros, J. Arsuaga, M. E. Vazquez, D. W. Sumners, J. Roca. Novel display of knotted DNA molecules by two-dimensional gel electrophoresis. Nucleic Acids Res. 29 (2001), E677.

[34] J. L. Nitiss. Targeting DNA topoisomerase II in cancer chemotherapy. Nat Rev Cancer. 9 (2009), 338-350.

[35] K. D. Corbett, J. M. Berger. Structure, molecular mechanisms, and evolutionary relationships in DNA topoisomerases. Annu. Rev. Biophys. Biomol. Struct. 33 (2004), 95-118. 
[36] P. Forterre, D. Gadelle. Phylogenomics of DNA topoisomerases: their origin and putative roles in the emergence of modern organisms. Nucleic Acids Research. 37 (2009), 679-692.

[37] J. M. Berger, S. J. Gamblin, S. C. Harrison, J. C. Wang. Structure and mechanism of DNA topoisomerase II. Nature. 379 (1996), 225-232.

[38] C. A. Austin, L. M. Fisher. DNA topoisomerases: enzymes that change the shape of DNA. Sci. Prog. 74 (1990), 147-161.

[39] J. J. Champoux. DNA topoisomerases: structure, function, and mechanism. Annu. Rev. Biochem. 70 (2001), 369-413.

[40] J. Roca. The mechanisms of DNA topoisomerases. Trends Biochem. Sci. 20 (1995), 156-160.

[41] F. R. Blattner, G. Plunkett III, C. A. Bloch, N. T. Perna, V. Burland, M. Riley, J. ColladoVides, J. D. Glasner, C. K. Rode, G. F. Mayhew, J. Gregor, N. W. Davis, H. A. Kirkpatrick, M. A. Goeden, D. J. Rose, B. Mau, Y. Shao. The complete genome sequence of Escherichia coli K-12. Science. 277 (1997), 1453-1462.

[42] P. H. von Hippel, E. Delagoutte. Macromolecular complexes that unwind nucleic acids. Bioessays. 25 (2003), 1168-1177.

[43] A. Worcel, S. Strogatz, D. Riley. Structure of chromatin and the linking number of DNA. Proc. Natl. Acad. Sci. USA 78 (1981), 1461-1465.

[44] L. A. A. Nicholl, D. S. T. Nicholl. Modelling the Eukaryotic chromosome: A stepped approach. Journal of Biological Education. 21 (1987), 99-103.

[45] E. Lieberman-Aiden, N. L. van Berkum, L. Williams, M. Imakaev, T. Ragoczy, A. Telling, I. Amit, B. R. Lajoie, P. J. Sabo, M. O. Dorschner, R. Sandstrom, B. Bernstein, M. A. Bender, M. Groudine, A. Gnirke, J. Stamatoyannopoulos, L. A. Mirny, E. S. Lander, J. Dekker. Comprehensive mapping of long-range interactions reveals folding principles of the human genome. Science. 326 (2009), 289-293.

[46] A. Y. Grosberg, S. K. Nechaev, E. I. Shakhnovich. The role of topological constraints in the kinetics of collapse of macromolecules. J.Phys.France. 49 (1988), 2095-2100.

[47] M. Buenemann, P. Lenz. A geometrical model for DNA organization in bacteria. PLoS ONE. 5 (2010), e13806.

[48] T. A. Shapiro, P.T. Englund. The structure and replication of kinetoplast DNA. Annu. Rev. Microbiol. 49 (1995), 117-143.

[49] J. Chen, C. A. Rauch, J. H. White, P. T. Englund, N. R. Cozzarelli. The Topology of the Kinetoplastic DNA nework. Cell, 80 (1995), 61-69. 
[50] J. Lukes, D. Lys Guilbride, J. Votypka, A. Zikova, R. Benne, P. T. Englund. Kinetoplast DNA network: evolution of an improbable structure. Eukaryotic Cell. 1 (2002), 495-502.

[51] G. W. Hatfield, C. J. Benham. DNA topology-mediated control of global gene expression in Escherichia coli. Annu. Rev. Genet. 36 (2002), 175-203.

[52] A. Vologodskii, N. R. Cozzarelli. Effect of supercoiling on the juxtaposition and relative orientation of DNA sites. Biophys. J. 70 (1996), 2548-2556.

[53] V. V. Rybenkov, C. Ullsperger, A. V. Vologodskii, N. R. Cozzarelli. Simplification of DNA topology below equilibrium values by type II topoisomerases. Science. 277 (1997), 690-693.

[54] A. Vologodskii. Theoretical models of DNA topology simplification by type IIA DNA topoisomerases. Nucleic Acids Res. 37 (2009), 3125-3133.

[55] K. C. Neuman. Single-molecule measurements of DNA topology and topoisomerases. J. Biol. Chem. 285 (2010), 18967-18971.

[56] N. Sonnenschein, M Geertz, G. Muskhelishvili, M. Hütt. Analog regulation of metabolic demand. MBC Systems Biology 5 (2011), 40-52.

[57] R. Messer, P. Staffin. Topology now! Math Assoc. of America, 2006. 